\title{
Inertial and buoyancy effects on the flow of elongated bubbles in horizontal channels
}

\author{
H.R. Moran ${ }^{1,2}$, M. Magnini ${ }^{3}$, C.N. Markides² ${ }^{2}$ and O.K. Matar ${ }^{1 *}$
}

This is the final accepted version of the manuscript. The final published version can be found at: https://doi.org/10.1016/j.ijmultiphaseflow.2020.103468

${ }^{1}$ Department of Chemical Engineering, Imperial College London, South Kensington Campus, London SW7 2AZ, United Kingdom

${ }^{2}$ Clean Energy Processes (CEP) Laboratory, Department of Chemical Engineering, Imperial College London, South Kensington Campus, London SW7 2AZ, United Kingdom

${ }^{3}$ Department of Mechanical, Materials and Manufacturing Engineering, University of Nottingham, Nottingham, NG7 2RD, United Kingdom

* Corresponding author. Email: o.matar@imperial.ac.uk

\begin{abstract}
When a long gas bubble travels in a horizontal liquid-filled channel of circular crosssection, a liquid film is formed between the bubble and the channel wall. At low Reynolds and Bond numbers, inertial and buoyancy effects are negligible, and the liquid film thickness is a function of the capillary number only. However, as the tube diameter is increased to the millimetre scale, both buoyancy and inertial forces may become significant. We present the results of a systematic analysis of the bubble shape, inclination, and liquid film thickness for a wide range of capillary, Bond, and Reynolds numbers, namely $0.024 \leq \mathrm{Ca}_{1} \leq 0.051$, $0.11 \leq \mathrm{Bo} \leq 3.5$, and $1 \leq \operatorname{Re}_{1} \leq 750$. Three-dimensional numerical simulations of the flow are performed by employing the Volume-Of-Fluid method implemented in OpenFOAM. In agreement with previous studies, we observe that buoyancy lifts the bubble above the channel axis, making the top liquid film thinner, and thickening the bottom film. As the Bond number approaches unity, the cross-sectional shape of the bubble deviates significantly from a circular shape, due to flattening of the bottom meniscus. The simulations demonstrate the existence of a cross-stream film flow that drains liquid out of the top film and drives it towards the bottom film region. This drainage flow causes inclination of the bubble, with a larger inclination angle along the bottom plane of the bubble than the top. As buoyancy becomes even more significant, draining flows become less effective and the bubble inclination reduces. A theoretical model for the liquid film thickness and bubble speed is proposed embedding dependencies on both capillary and Bond numbers, which shows good agreement with the reported numerical results. Inertial forces tend to shrink the bubble cross-section and further lift the bubble above the channel centreline, so that the bottom film thickness increases significantly with the Reynolds number, whereas the top film thickness is less sensitive to it.
\end{abstract}

Keywords: Elongated bubble; buoyancy; inertia; two-phase; bubble asymmetry; liquid film.

\section{Introduction}

Elongated bubble flow in liquid-filled channels is relevant to a wide variety of industries, being of interest in a range of heat and mass transfer as well as lubrication problems. The research 
on elongated bubble flow problems is motivated by applications such as treatment of pulmonary disorders, enhanced oil recovery (Magnini et al. 2017a), boiling flows in refrigeration (Karayiannis and Mahmoud 2017) and concentrating solar power (Dirker et al. 2019). In such flows, an elongated bubble travels at a speed $U_{\mathrm{b}}$ in a channel in which liquid flows at an average velocity $U_{\mathrm{l}}$ (with $U_{\mathrm{b}}>U_{\mathrm{l}}$ ), and has density, viscosity and surface tension of $\rho_{\mathrm{l}}, \mu_{\mathrm{l}}$, and $\sigma$, respectively. The flow can be characterised by the following dimensionless numbers: the Reynolds number $\operatorname{Re}=\rho_{\mathrm{l}} U D / \mu_{\mathrm{l}}$, where $D$ is the diameter of the circular cross-section of the channel with corresponding radius $R=D / 2$, which describes the relative magnitudes of the inertial and viscous forces; the capillary number $\mathrm{Ca}=\mu_{1} U / \sigma$, which describes the relative magnitude of the viscous and interfacial tension forces; and the Bond number $\mathrm{Bo}=\rho_{\mathrm{l}} g R^{2} / \sigma$, where $\mathrm{g}$ is the gravitational acceleration, which describes the relative magnitude of the buoyancy and interfacial tension forces. Elongated bubble flow has been widely studied in microchannels, where inertial and buoyancy effects are often negligible, i.e. $(\mathrm{Bo}, \mathrm{Re}) \ll 1$. In this regime, an axisymmetric liquid film separates the bubble from the tube wall. Bretherton (1961) developed the classical theoretical prediction of the thickness of this liquid film, applying lubrication theory to find that, for flows where $\left(\mathrm{Ca}_{\mathrm{b}}, \mathrm{Re}_{\mathrm{b}}\right) \ll 1$ (and neglecting buoyancy effects) the liquid film thickness $h$ scales with $\mathrm{Ca}_{\mathrm{b}}{ }^{2 / 3}$ as follows:

$$
H=h / R=1.34 \mathrm{Ca}_{\mathrm{b}}{ }^{2 / 3}
$$

where $H$ is the film thickness non-dimensionalised by the characteristic length of the channel cross-section, $R$ for a circular capillary. Taylor (1961) carried out experiments at higher $\mathrm{Ca}_{\mathrm{b}}$ using very viscous fluids and found that as $\mathrm{Ca}_{\mathrm{b}} \rightarrow 2, H$ approaches an asymptotic value of approximately $1 / 3$.

Various improvements to the Bretherton model have been suggested to expand its range of applicability. Aussillous and Quéré (2000) showed that the validity of Eq. (1) could be extended to larger capillary numbers $\left(\mathrm{Ca}_{\mathrm{b}} \approx 1\right)$ by replacing $R$ with $R-c h$, where $c$ is an empirical constant, to account for the effect of a thick film on the curvature of the front meniscus of the bubble. The modified film thickness relationship compared well with their experimental liquid film thickness data when setting $c=2.5$, giving the following equation:

$$
H=\frac{1.34 \mathrm{Ca}_{\mathrm{b}}^{2 / 3}}{1+3.35 \mathrm{Ca}_{\mathrm{b}}^{2 / 3}}
$$

For finite $\mathrm{Re}_{\mathrm{b}}$, inertial effects become significant and a modification to Eq. (2) that takes these into account is required. The impact of inertia on liquid film thickness in micro-tubes was investigated numerically and experimentally by Kreutzer et al. (2005), whose results showed a similar trend to the numerical results of Heil (2001). Han and Shikazono (2009) performed experiments at higher $\mathrm{Ca}_{\mathrm{b}}$ and $\mathrm{Re}_{\mathrm{b}}$, observing that at these conditions the effect of inertia cannot be neglected. They applied a scaling analysis to obtain a relation of the following form for $\operatorname{Re}_{\mathrm{b}}<2000$ :

$$
H=\frac{1.34 \mathrm{Ca}_{\mathrm{b}}{ }^{2 / 3}}{1+3.35 \mathrm{Ca}_{\mathrm{b}}{ }^{2 / 3}+f\left(\mathrm{Ca}_{\mathrm{b}}, \mathrm{Re}_{\mathrm{b}}\right)-g\left(\mathrm{We}_{\mathrm{b}}\right)}
$$

where $\mathrm{We}_{\mathrm{b}}$ is the bubble Weber number, defined as $\mathrm{We}_{\mathrm{b}}=\rho U_{\mathrm{b}}^{2} D / \sigma=\mathrm{Re}_{\mathrm{b}} \mathrm{Ca}_{\mathrm{b}}$. They obtained empirical coefficients through fitting of experimental data and reported film thickness predictions with $\pm 15 \%$ accuracy. The experimental and numerical results of Khodaparast et al. (2015) exhibited close agreement to those of Han and Shikazono (2009).

The studies above were all conducted for micro-tubes and therefore did not consider the effect of buoyancy, that may become impactful already for tubes of diameters slightly above $1 \mathrm{~mm}$ or Bo $\ll 1$ (Magnini et al. 2019, Hazel and Heil 2002). Intuition suggests that, in a horizontal tube, buoyancy acts by elevating the bubble above the channel centreline, thus developing a circumferential asymmetry of the liquid film, where the top film becomes thinner than the 
bottom film; see the schematic in Fig. 1. Jensen et al. (1987) studied the effect of gravity on the propagation of an air finger within a horizontal Hele-Shaw cell. By applying a theoretical analysis based on matched asymptotic expansions in the small capillary number limit, $\mathrm{Ca}_{\mathrm{b}} \lesssim 10^{-3}$, they derived the following leading-order relationship for the top and bottom liquid film thicknesses $\left(h^{+}\right.$and $h^{-}$, respectively) when Bo $<1$ :

$$
\frac{h^{ \pm}}{b}=\frac{1.34 \mathrm{Ca}_{\mathrm{b}}{ }^{2 / 3}}{1 \pm \mathrm{Bo}}
$$

where $b$ is half the thickness of the Hele-Shaw cell, which corresponds to the pipe radius $R$ in the present case. For vanishing values of the Bond number, Eq. (4) simplifies to Eq. (1) and as Bo increases, the top film thickness $\left(h^{+}\right)$decreases and the bottom one $\left(h^{-}\right)$increases. Note that Eq. (4) is not applicable to estimate $h^{-}$when Bo $\rightarrow 1^{-}$, as predictions diverge to $+\infty$. When Bo $>1$, Jensen et al. (1987) pointed out that the hydrostatic pressure in the bottom film becomes larger than the pressure induced by viscous forces. In this regime, the bubble detaches from the bottom wall, so that a wetting film is present only at the top, while the bubble thickness becomes independent of the height of the channel. Using their leading-order relationships for the thicknesses of the bubble and the top film, the following expressions can be written:

$$
\begin{aligned}
\frac{h^{+}}{b} & =\frac{1.34 \mathrm{Ca}_{b}^{2 / 3}}{2 \sqrt{\mathrm{Bo}}} \\
\frac{h^{-}}{b} & =2 \frac{\sqrt{\mathrm{Bo}}-1}{\sqrt{\mathrm{Bo}}}
\end{aligned}
$$

which suggest that $h^{+} \rightarrow 0$ and $h^{-} \rightarrow 2 b$ as Bo $\rightarrow \infty$, i.e. the top film vanishes and the bottom film thickness converges to the Hele-Shaw cell thickness. Note that, as Bo $\rightarrow 1^{+}$, Eq. (5a) converges smoothly to Eq. (4), whereas $h^{-} \rightarrow 0$. Therefore, while Eqs. (4) and (5a) yield continuous predictions of the top film thickness as Bo approaches 1 from both left and right, predictions of the bottom film thickness from Eqs. (4) and (5b) diverge at Bo $=1$. In deriving the equations above, Jensen et al. (1987) assumed that $h \ll b$, such that the radius of the bubble front cap could be approximated as $b$, which is an acceptable assumption as long as $\mathrm{Ca}_{\mathrm{b}} \lesssim 10^{-3}$ (Aussillous and Quéré 2000). Atasi et al. (2017) accounted for the presence of thick top and bottom films, to derive better matching conditions for the film profile at the bubble nose, and thus extend the applicability of the model of Jensen et al. (1987) to larger $\mathrm{Ca}_{\mathrm{b}}$. The resulting predictive method was a four-equation model, valid for $\mathrm{Bo}<1$, requiring an iterative solution, which they compared against experimental data for long bubbles travelling in a very viscous liquid $\left(\mathrm{Re}_{\mathrm{b}} \ll 1\right)$ in horizontal tubes with $0.05<\mathrm{Bo}<0.4$ and $10^{-3}<\mathrm{Ca}_{\mathrm{b}}<10^{-1}$. The comparison was satisfactory at low Bo, whereas the increasing deviations at larger Bo were attributed to the ovalisation of the cross-sectional shape of the bubble, which could not be accounted for in their two-dimensional flow model.

Leung et al. (2012) measured top and bottom film thicknesses and liquid flow field for long gas bubbles travelling in horizontal channels in the range $\mathrm{Bo} \leq 0.25,0.05 \leq \mathrm{Ca}_{\mathrm{b}} \leq 0.2$, and weak inertia effects $\left(\operatorname{Re}_{\mathrm{b}} \leq 50\right)$. Besides the asymmetry in the top and bottom liquid film heights, they observed that the bubble was inclined with respect to the tube axis (see Fig. 1), and related it to the drainage of the top film as liquid flows downward under the action of gravity. Assuming negligible deformation of the bubble from a circular shape in the cross-section, they applied a mass balance to the draining film flow and derived an expression for the bubble inclination, which Atasi et al. (2017) rearranged in the following form:

$$
\tan \alpha=\frac{\left(H^{\mathrm{h}}\right)^{3}}{3 \mathrm{Ca}_{\mathrm{b}}} \text { Bo }
$$

where $\alpha$ is the inclination angle and $H^{\mathrm{h}}$ is the dimensionless film thickness extracted at the horizontal centreplane of the channel. It should be noted that the original theory and Eq. (6) 


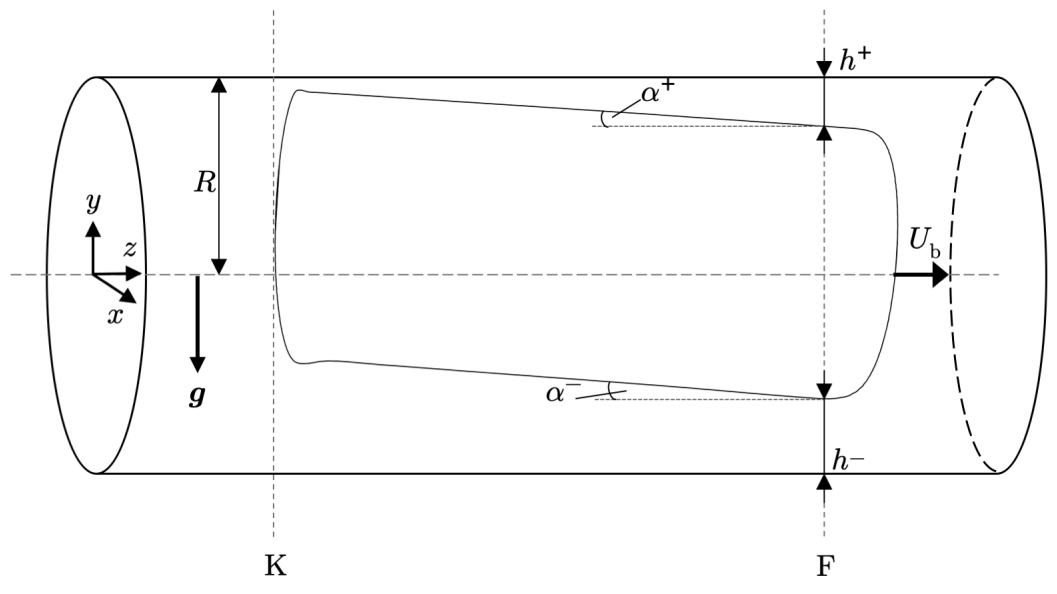

(a)

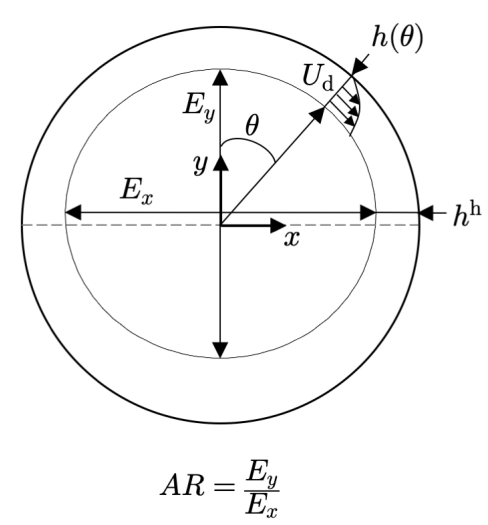

(b)

Figure 1: Sketch of an elongated gas bubble flowing in a horizontal tube filled with liquid, and notation used in this work. (a) Two-dimensional bubble profile extracted on a vertical centreline plane, where $x=0$. (b) Bubble cross-sectional profile extracted on an arbitrary $x-y$ plane. In (a), position ' $\mathrm{K}$ ' indicates the most upstream point of the bubble interface; position ' $\mathrm{F}$ ' identifies the location of the lowest point of the bubble interface, which in this work is selected as the location where the film thicknesses are measured.

do not distinguish between top and bottom inclination angles. However, Atasi et al. (2017) observed experimentally that the inclination angle at the top of bubble was smaller than that at the bottom, and compared the predictions of Eq. (6) to an average experimental inclination angle with good agreement between the two.

The literature survey presented above emphasises that a systematic analysis of the liquid film topology and thickness for long bubbles travelling in horizontal tubes at Bo $\geq 1$, and non-neglibile inertial effects, is still missing. This work uses direct numerical simulations to investigate systematically the effects of buoyancy and inertia on elongated bubble flows for the parameter range $0.024 \leq \mathrm{Ca}_{1} \leq 0.051,0.11 \leq \mathrm{Bo} \leq 3.5$, and $1 \leq \mathrm{Re}_{1} \leq 750$. Simulations of the immiscible flow of liquid and gas phases are run using the open-source package OpenFOAM (ESI; v. 1606) and its built-in Volume-Of-Fluid (VOF) method (Hirt and Nichols 1981). The use of a three-dimensional model enables insights into the impact of buoyancy and inertia on the shape of the bubble cross-section, a view that is difficult to obtain experimentally. A description of the problem in question and the corresponding numerical model are provided in Section 2 , A theoretical predictive method for the top and bottom liquid film thicknesses with finite Bo is proposed in Section 3, whilst the numerical results are presented and discussed in Section 4. In Section 5, the main conclusions are summarised.

\section{Numerical model}

\subsection{Problem description}

We consider an elongated bubble travelling at a velocity $U_{\mathrm{b}}$ along a horizontal channel of circular cross-section with radius $R$ (diameter $D$ ), through which liquid flows at an average velocity $U_{1}$. A representative sketch of the flow problem is shown in Fig. 1. The coordinate $z$ is directed downstream, whilst the coordinates $x$ and $y$ represent the horizontal and vertical axes, respectively, with the origin located at the centre of the channel. The flow is subject to a gravitational acceleration, $\mathbf{g}$, acting in the negative $y$-direction. 
In the absence of buoyancy effects, a region of constant film thickness between the bubble nose and tail exists. However, this does not happen in the case of non-negligible buoyancy effects, because the bubble is inclined in the channel (Atasi et al. 2017; Leung et al. 2012). Therefore, the film thicknesses reported in this paper are extracted not at the front of the bubble, but behind the bubble nose to ensure film development. This location is given the signifier ' $\mathrm{F}$ ' and is defined as the $z$-value at which the minimum film thickness around the lower half of the bubble, $h^{-}$, occurs, with a corresponding film thickness at the top of the bubble $h^{+}$; see Fig. 1. The film thickness is non-dimensionalised with the channel radius $R$, such that $H=h / R$, and all subsequent film thicknesses in this paper are reported as dimensionless values $\left(H^{+}\right.$and $\left.H^{-}\right)$at location $\mathrm{F}$. The inclination angles at the top and bottom of the bubble, $\alpha^{+}$and $\alpha^{-}$are calculated by considering the slopes of the developed middle regions of the bubble profile upstream of the nose but downstream of the tail, quantified as the region $2 D$ up- and downstream of the centre of the bubble lengthwise.

The back of the bubble, location $\mathrm{K}$, is defined as the minimum $z$-value in the bubble profile. Hereafter, the $z$-dimension will be non-dimensionalised in the manner $\left(z-z_{\mathrm{K}}\right) / R$, thus always referring to coordinates relative to the tip of the bubble tail. The bubble aspect-ratio in the cross-section, $A R$, describes the ovalisation of the bubble on the $x-y$ plane and is defined as $A R=E_{y} / E_{x} ; E_{y}$ is the maximum extension of the bubble along the vertical direction, which always occurs along the $y$-axis; $E_{x}$ is the maximum extension of the bubble along the horizontal direction, and this location shifts above the $x$-axis as buoyancy effects become important (see Fig. 1 b) and the whole bubble elevates above the channel axis. Note that $A R=1$ corresponds to an exact axisymmetric bubble (i.e. negligible gravity effects), whereas buoyancy tends to reduce the vertical extension of the bubble, leading to $A R<1$.

Besides the top and bottom film thicknesses (both evaluated along the vertical $x=0$ axis), we identify also a horizontal film thickness $h^{h}$, which is defined as the horizontal distance between the bubble interface and the tube wall, calculated along the same line where the maximum bubble extension $E_{x}$ is identified, see Fig. $1 \mathrm{~b}$. Figure $1 \mathrm{~b}$ also includes a schematic representing the velocity profile in the liquid film region, on the cross-section of the channel. Along a radial line extending from the liquid-gas interface to the pipe wall, where the film has thickness $h(\theta)$, the velocity profile is parabolic due to the no-slip condition at the wall and the negligible tangential stress exerted by the gas phase at the interface. The average velocity of the liquid in the film, obtained by integrating this velocity profile, is denoted as $U_{\mathrm{d}}$ and is a function of the circumferential angle, $U_{\mathrm{d}}(\theta)$. In this work, $h^{h}, A R$ and $U_{\mathrm{d}}$ are always extracted on a cross-section located at $\mathrm{F}$.

The flow is assumed to be laminar and incompressible, and the two phases Newtonian and immiscible. The Reynolds number is defined as $\operatorname{Re}=\rho U D / \mu$ where the density $\rho$ and the dynamic viscosity $\mu$ are those of the liquid, but the velocity $U$ can be that of the bubble $\left(U_{\mathrm{b}}\right)$ or liquid $\left(U_{1}\right)$. The capillary number is similarly defined as $\mathrm{Ca}=\mu U / \sigma$, where $\sigma$ is the interfacial tension. The Weber number is then defined as $\mathrm{We}=\mathrm{Re} \mathrm{Ca}=\rho U^{2} D / \sigma$. The results in this study are mostly reported as functions of the liquid dimensionless numbers, since simulations can be easily initialised for fixed values of these and in experimental studies of isolated bubble flow the liquid flow rate is usually the controlled parameter. The buoyancy effects are characterised by the Bond number, Bo $=\left(\rho_{\mathrm{l}}-\rho_{\mathrm{g}}\right) g R^{2} / \sigma \simeq \rho_{\mathrm{l}} g R^{2} / \sigma$, where the gas density is assumed to be negligible compared to the liquid.

\subsection{Numerical method}

The numerical framework is based on OpenFoam's built-in VOF solver interFoam (Deshpande et al. 2012). This solves the single-fluid continuity and momentum conservation equations for a Newtonian fluid in incompressible flow in the following form:

$$
\nabla \cdot \mathbf{u}=0
$$




$$
\frac{\partial(\rho \mathbf{u})}{\partial t}+\nabla \cdot(\rho \mathbf{u u})=-\nabla p+\nabla \cdot \mu\left[(\nabla \mathbf{u})+\left(\nabla \mathbf{u}^{T}\right)\right]+\rho \mathbf{g}+\mathbf{F}_{\sigma}
$$

where $\mathbf{u}$ is the fluid velocity vector, $\mathbf{g}$ is the gravitational acceleration vector, and $\mathbf{F}_{\sigma}$ is the surface tension force. The fluid properties $\rho$ and $\mu$ are defined as average properties weighted by the volume fraction $\alpha$ as follows:

$$
\begin{aligned}
& \rho=\rho_{1} \alpha+\rho_{2}(1-\alpha) \\
& \mu=\mu_{1} \alpha+\mu_{2}(1-\alpha)
\end{aligned}
$$

where the subscripts 1 and 2 denote the primary and secondary phases, respectively. $\alpha$ is defined as the ratio of the cell volume occupied by the primary phase, so is equal to 1 in a cell filled with the primary phase and 0 in a cell filled with the secondary phase, and is in the range $0<\alpha<1$ for an interfacial cell containing both phases. The fluid properties $\rho_{1}, \mu_{1}, \rho_{2}$ and $\mu_{2}$ are considered constant in this work. The surface tension force $\mathbf{F}_{\sigma}$ in Eq. (8) is formulated as a body force according to the continuum surface force (CSF) method proposed by Brackbill et al. (1992) as $\mathbf{F}_{\sigma}=\sigma \kappa \nabla \alpha$, where the surface tension coefficient $\sigma$ is considered to be constant and $\kappa$ is the local interface curvature. The latter is estimated by means of derivatives of the volume fraction field, $\kappa=-\nabla \cdot \mathbf{n}$, where $\mathbf{n}$ is the interface unit normal vector, $\mathbf{n}=\nabla \alpha /|\nabla \alpha|$.

InterFoam solves the volume fraction equation by including an artificial compressive term to counteract numerical diffusion at the interface:

$$
\frac{\partial \alpha}{\partial t}+\nabla \cdot(\alpha \mathbf{u})+\nabla \cdot\left(\alpha(1-\alpha) \mathbf{U}_{\mathbf{r}}\right)=0
$$

where the artificial compressive velocity $\mathbf{U}_{\mathbf{r}}$ is given by:

$$
\mathbf{U}_{\mathbf{r}}=\min \left[C_{\alpha} \frac{|\phi|}{\left|\mathbf{S}_{\mathbf{f}}\right|},\left(\frac{|\phi|}{\left|\mathbf{S}_{\mathbf{f}}\right|}\right)_{\max }\right] \mathbf{n}_{\mathbf{f}}
$$

Here, $\phi$ is the volumetric flux through the control volume face, $\mathbf{S}_{\mathbf{f}}$ is the surface area vector of the cell face, $\mathbf{n}_{\mathbf{f}}$ is the unit normal vector to the interface evaluated at the control volume face, and $C_{\alpha}$ is a numerical multiplier used for tuning of the interface compression. For this study, the default value $C_{\alpha}=1$ (Khodaparast et al. 2015 Ferrari et al. 2017; Deshpande et al. 2012) has been used since it presents an appropriate compromise between interface sharpness and accuracy of the surface tension calculation.

The flow equations are discretised based on a finite-volume discretisation in a co-located grid arrangement. Eq. (11) is discretised based on the Multidimensional Universal Limiter with Explicit Solution (MULES) algorithm, a first-order time-explicit scheme. The temporal discretisation of the remaining equations follows a first-order implicit scheme. The divergence terms are discretised with second-order upwind schemes, whereas the Laplacian terms utilise second-order central finite-difference schemes. The pressure and velocities are iteratively updated within each time step using the Pressure Implicit Splitting of Operators (PISO) algorithm (Issa 1986), with the number of correctors set to 3. The residual thresholds for the iterative solution of the flow equations are set to $10^{-6}$ for velocity, $10^{-8}$ for volume fraction, and $10^{-7}$ for pressure. A variable time-step is adopted for the progress of the solution, the value of which is calculated by the solver in accordance with an assigned maximum Courant number of Co $=0.4$.

\subsection{Simulation set-up}

The simulated geometry consists of a channel of circular cross-section with radius $R$, modelled with a 3-D mesh, through which liquid flows at an average velocity $U_{1}$. A channel length of $100 R$ is simulated to allow for full development of the flow. At the inlet, a uniform liquid velocity 


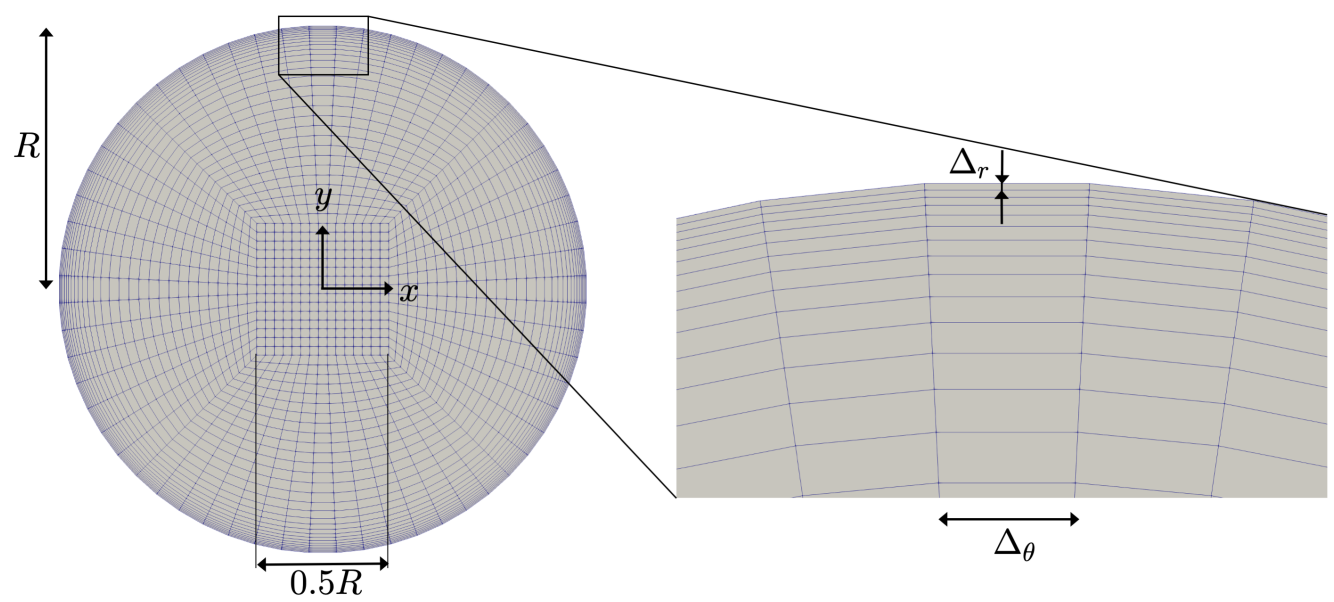

Figure 2: Illustration of the $x-y$ cross-section of the computational mesh. The core consists of a square rod of size $0.5 R \times 0.5 R$ meshed with uniform cubes, outside of which the mesh is gradually refined radially towards the wall. At the wall, the size of the elements along the radial and circumferential directions are denoted as $\Delta_{r}$ and $\Delta_{\theta}$, respectively. For the mesh depicted here, $\Delta_{r}=0.0041 R$ and $\Delta_{\theta}=0.105 R$.

profile is fixed, along with a zero-gradient pressure condition. At the outlet, the velocity is subject to a zero-gradient condition whilst the pressure is set to a uniform reference value. Noslip is imposed at the walls along with a zero-gradient pressure condition. A bubble is initiated close to the inlet at the centre of the channel with a radius of $0.9 R$ and a length of $20 R$ to ensure full development of the liquid film between the nose and tail across the whole parameter space (Magnini et al. 2017b). The liquid to gas density and viscosity ratios are set to 1000 and 100, respectively.

The computational grid, the $x-y$ cross-section of which is depicted in Fig. 2, is a blockstructured mesh. The core of the domain is discretised with a uniform mesh made of cubes. Outside of this central core, the mesh is refined radially towards the wall, allowing capture of liquid film dynamics without the computational expense of a universally fine mesh. For accurate simulations of liquid films, Gupta et al. (2009) recommended that there should be at least 5 cells in the liquid film in the radial direction. To determine the optimum mesh configuration, a preliminary grid convergence study was performed, starting with the conditions $\mathrm{Ca}_{1}=0.024$, Bo $=0.42$, and $\operatorname{Re}_{1}=1$, characterised by a minimum film thickness at the bubble top of $H^{+} \approx 0.07$. Converged results were obtained by employing a mesh with the finest element at the wall of $\Delta_{r}=0.0041 R$, which is the mesh displayed in Fig. 2. The same mesh was then used for conditions characterised by similar values of the Bond number, whereas a much finer mesh $\left(\Delta_{r}=0.0023 R\right)$ was found necessary for larger Bond numbers, where the film becomes as thin as $H^{+} \approx 0.03$. The Reynolds number has only a minor impact on the film thickness, and hence the mesh utilised for $\mathrm{Re}_{\mathrm{l}}=1$ was found to be suitable also for larger values of $\mathrm{Re}_{\mathrm{l}}$. The full details of the mesh convergence study are reported in the Supplementary Material.

In the validation Section 4.1, the results of 2-D simulations, with the gravitational force vector acting transversal to the flow direction, will be presented together with the 3-D cases. The 2-D set-up and boundary conditions are analogous to the $3-\mathrm{D}$, and an orthogonal mesh with near-wall refined elements is employed to have a minimum of 10 cells resolving the flow in the thin liquid film. 


\section{Theoretical predictions}

In this section, we review and further develop existing predictive models for the liquid film thickness, bubble speed and liquid drainage velocities that will be useful as a benchmark for the computational data presented in the next section.

\subsection{Liquid film thickness}

The equations derived theoretically by Jensen et al. (1987), Eqs. (4), (5a), and (5b), apply as long as $\mathrm{Ca}_{\mathrm{b}} \lesssim 10^{-3}$. In order to extend their validity to large capillary numbers, we follow the approach of Aussillous and Quéré (2000) and assume that the radius of curvature of the bubble nose on the top/bottom of the channel is reduced from $R$ to $R-2.5 h^{ \pm}$to account for the presence of a thick film. This modifies Eq. (4), valid for Bo $<1$, into:

$$
\frac{h^{ \pm}}{R}=\frac{1.34 \mathrm{Ca}_{\mathrm{b}}{ }^{2 / 3}}{1+3.35 \mathrm{Ca}_{\mathrm{b}}{ }^{2 / 3} \pm \mathrm{Bo}}
$$

and Eq. (5a), valid for the top film thickness when Bo > 1, into:

$$
\frac{h^{+}}{R}=\frac{1.34 \mathrm{Ca}_{\mathrm{b}}{ }^{2 / 3}}{2 \sqrt{\mathrm{BO}}+3.35 \mathrm{Ca}_{\mathrm{b}}{ }^{2 / 3}}
$$

Note that the two equations above yield predictions for the top film thickness that converge at $\mathrm{Bo}=1$. On the other hand, Eq. (13) for the bottom film thickness diverges to $\infty$ as $\mathrm{Bo} \rightarrow 1+3.35 \mathrm{Ca}_{\mathrm{b}}{ }^{2 / 3}$, and therefore it should not be used when Bo is close to unity.

The correction of Eq. (5p) to account for a thick film at the bottom of the channel is done by replacing $R$ ( $b$ in Eq. $(5 b)$ ) with $R-2.5 h_{A Q}$, where $h_{A Q}$ is the film thickness in the absence of gravitational effects estimated via the Aussillous and Quéré (2000) correlation (Eq. (2)), thus leading to:

$$
\frac{h^{-}}{R}=2 \frac{\sqrt{\mathrm{Bo}}-1}{\sqrt{\mathrm{Bo}}\left(1+3.35 \mathrm{Ca}_{\mathrm{b}}^{2 / 3}\right)}
$$

This introduces the effect of the capillary number in the prediction of the bottom film thickness for $\mathrm{Bo}>1$, which was otherwise absent in Eq. (5b). Note that Eq. (15) yields $h^{-} \rightarrow 0$ as Bo $\rightarrow 1^{+}$, and therefore it should not be used for Bond numbers close to unity. In Section 4.2.2. the liquid film thickness predictions extracted from the set of equations above will be compared with the simulation data.

\subsection{Bubble advancing speed and liquid drainage velocities}

A useful relationship linking liquid film thickness, streamwise bubble and liquid average velocities is provided by a material balance of the liquid in a control-volume containing the bubble (Bretherton 1961). In a reference frame moving with the bubble, the liquid displaced by the advancing bubble must match the liquid bypassing the bubble through the liquid film, thus leading to:

$$
\frac{U_{\mathrm{b}}}{U_{\mathrm{l}}}=\frac{1}{(1-H)^{2}}
$$

where $H=h / R$ is the axisymmetric film thickness, or a cross-sectional average film thickness $\bar{H}$ in the case of asymmetric film. In the latter case, $\bar{H}$ can be estimated as $\bar{H}=\left(H^{+}+H^{-}+2 H^{\mathrm{h}}\right) / 4$, with $H^{+}$from Eq. 13) $($Bo $<1)$ or Eq. (14) $($ Bo $>1), H^{-}$from Eq. 13) $($Bo $<1)$ or Eq. (15) $(\mathrm{Bo}>1)$, and the horizontal film thickness $H^{\mathrm{h}}$ with Aussillous and Quéré (2000) correlation, Eq. (2), under the assumption that the horizontal film thickness does not deviate significantly from the buoyancy-free configuration. In Section 4.2.2, the bubble to liquid speed ratio calculated 
from Eq. 16), with $\bar{H}$ either extracted from the numerical data or predicted as indicated above, will be compared with the simulation data.

For the cross-sectional liquid drainage velocity, i.e. the circumferential velocity of liquid averaged radially across the film at a certain angle $\theta$ (see Fig. 1b), Leung et al. (2012) proposed a fluid dynamic model based on the solution of the Navier-Stokes equations in the film. Under the assumption that the cross-section of the bubble remains circular, their model led to the following expression for the average drainage velocity $U_{\mathrm{d}}$ as a function of the circumferential position:

$$
U_{\mathrm{d}}(\theta)=\frac{\Delta \rho g h^{2}}{3 \mu}\left(1-\frac{3}{8} \frac{h}{R}\right) \sin \theta
$$

where $h \equiv h(\theta)$ and $\theta$ indicates the angle measured from the top. Note that $\theta=\pi / 2$ identifies the horizontal centreline of the cross-section (the $x$-axis in Fig. 1), irrespective of the actual bubble elevation above it. Equation (17) is rendered dimensionless by scaling $U_{\mathrm{d}}$ on $U_{\mathrm{l}}$ and by making use of the definitions of $\mathrm{Bo}$ and $\mathrm{Ca}_{1}$ :

$$
\frac{U_{\mathrm{d}}(\theta)}{U_{\mathrm{l}}}=\frac{1}{3} \frac{\mathrm{Bo}}{\mathrm{Ca}_{\mathrm{l}}} H^{2}\left(1-\frac{3}{8} H\right) \sin \theta
$$

where $H=h(\theta) / R$. Leung et al. (2012) simplified Eq. (18) by neglecting the cubic term and neglecting the circumferential changes of $H$, thus expressing $H$ as a function of the capillary number alone using Eq. (2). There seems to be an error in their final formula (Eq. 10 in Leung et al. (2012)), however, where the constant in the numerator should be 0.15 instead of 0.45. In Section 4.2.3, the predictions given by Eq. (18) will be benchmarked versus the present simulation results.

\section{Results}

In this section, we present the results of the numerical study of the effect of buoyancy and inertial forces on the dynamics of the bubble and of the liquid film surrounding it. The numerical model is first validated in Section 4.1. The effect of gravitational forces is then studied in Section 4.2 by systematically varying the Bond number, but fixing the Reynolds number to a small value, $\mathrm{Re}_{\mathrm{l}}=1$, such that the Weber number of the flow $\mathrm{We}_{1}=\mathrm{Ca}_{1} \mathrm{Re}_{1} \ll 1$. This enables us to isolate the effects of buoyancy from those of inertia. The analysis of the inertial regime is outlined in Section 4.3, where simulations were run by systematically varying the Reynolds number, for selected values of the Bond and capillary numbers.

\subsection{Validation}

Simulations were performed for elongated bubble flow in a horizontal tube at conditions designed to match those of the experiments by Atasi et al. (2017) $\left(\mathrm{We}_{1} \ll 1\right)$, with cases run in both 2 $\mathrm{D}$ and 3-D geometries to assess the most appropriate configuration. The experimental film thickness values are plotted against the results from both 2-D and 3-D simulations in Fig. 3 for $\mathrm{Bo}=0.11$ and 0.42 , along with the predictions obtained using Eq. (2), i.e. the Bo $=0$ case. For all of the plotted data sets, film thickness increases with $\mathrm{Ca}_{\mathrm{b}}$ as expected from traditional thin-film theory. The bottom film thickness $H^{-}$is larger than that at the top of the bubble $H^{+}$, because buoyancy causes the bubble to rise upwards in the channel. As Bo increases, $H^{+}$and $H^{-}$diverge more from the $\mathrm{Bo}=0$ theoretical prediction, with larger values of $H^{-}$and smaller values of $H^{+}$. It can be seen from Fig. 3 that the film thickness is significantly over-predicted by the 2-D simulations. It was also observed that bubbles were not inclined in the 2-D simulations, because bubble inclination is caused by film drainage from the top of the bubble around its sides to the bottom. This drainage flow is perpendicular to the main flow direction, so cannot be 


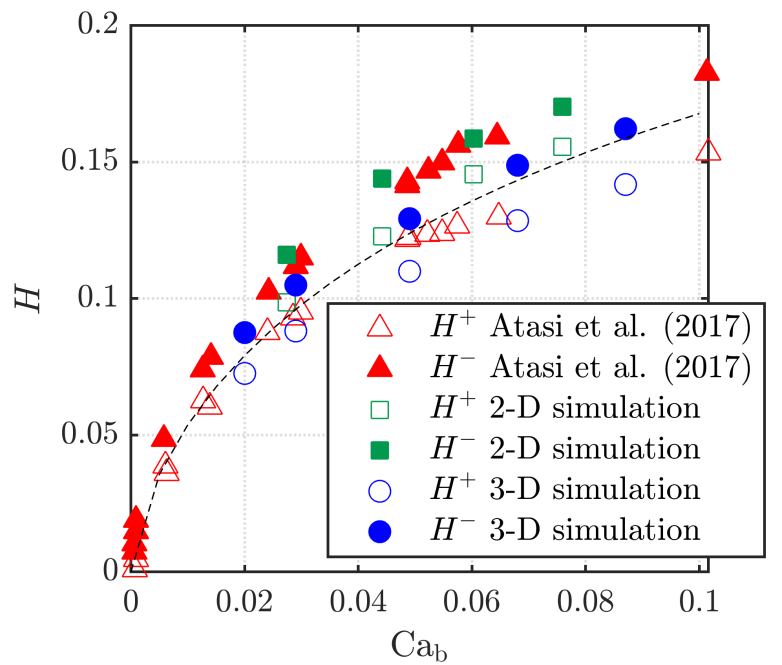

(a) $\mathrm{Bo}=0.11$

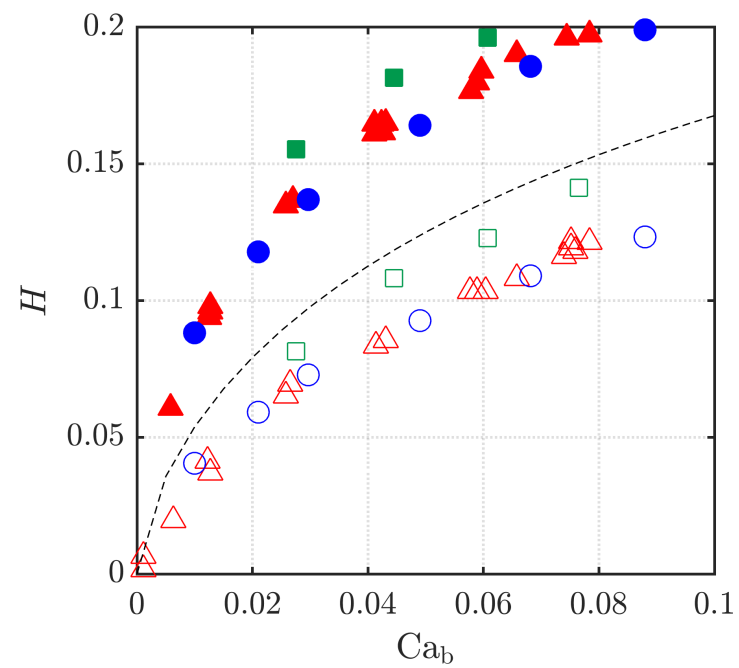

(b) $\mathrm{Bo}=0.42$

Figure 3: Comparison of the effect of the bubble capillary number and Bond number on the top and bottom film thicknesses with the experimental work of Atasi et al. (2017). The results of both 2-D and 3-D numerical simulations are reported. The dashed line represents the prediction of $H$ using the Aussillous and Quéré (2000) correlation, Eq. (2), i.e. in the Bo $=0$ case.

captured by a $2-\mathrm{D}$ simulation. Thus, whilst more computationally expensive, $3-\mathrm{D}$ simulations are required to accurately model elongated bubble flow in the presence of buoyancy effects.

The 3-D simulations match the experimental results very well, with a maximum deviation of $4 \%$ for $\mathrm{Bo}=0.42$ and $10 \%$ for $\mathrm{Bo}=0.11$, detected at lower values of $\mathrm{Ca}_{\mathrm{b}}$. Atasi et al. (2017) reported a maximum experimental error of $14 \%$ at the lowest $\mathrm{Ca}_{\mathrm{b}}$ and $5 \%$ for all other measurements, and the 3 -D computational results fall within these margins.

\subsection{Buoyancy effects}

A parametric study of the effect of buoyancy on elongated bubble flow with negligible inertia (i.e. $\mathrm{We}_{\mathrm{l}} \ll 1$ ) was performed for a range of $\mathrm{Ca}_{1}$ and Bo values, with results reported in this section for $\mathrm{Ca}_{1}=0.024$ and 0.051 and $\mathrm{Bo}=0.11$ to 3.5.

A representative case is visualised in Fig. 4, for $\mathrm{Ca}_{1}=0.024$ and $\mathrm{Bo}=0.42$. Three crosssections along the bubble, the locations of which are indicated on Fig. 4a, are shown in Fig. 4b d, The white space at the centre represents the volume occupied by the gas phase and the ovalisation of the bubble cross-section can be clearly seen. This is an important observation, since the crosssectional view of the bubble is very difficult to obtain experimentally. The coloured regions of Fig. $4 \mathrm{~b} d$ show the $y$-component of the velocity, $U_{y}$, in the liquid film, scaled by the average bubble velocity in the $z$-direction. It can be seen that $U_{y}$ is negative around the sides of the bubble, with the largest magnitude corresponding to the widest point of the bubble, confirming film drainage from the top to the bottom of the bubble as suggested by Leung et al. (2012). The magnitude of this drainage velocity is larger near the bubble nose and decreases towards the rear. This may be ascribed to the progressive thinning of the liquid film at the top and sides of the bubble as the rear is approached, which is apparent in Fig. 4b-d, which reduces the drainage flow rate.

The bubble inclination that is characteristic of buoyancy-affected elongated bubble flows can be clearly seen in Fig. 4e, along with well-defined rounded bubble nose and tail, which is typical in capillary flows. This figure is not to scale to allow for better visualisation of the aforementioned effects. The film thicknesses at location $\mathrm{F}$ for this case were calculated to be $H^{+}=0.073$ and 


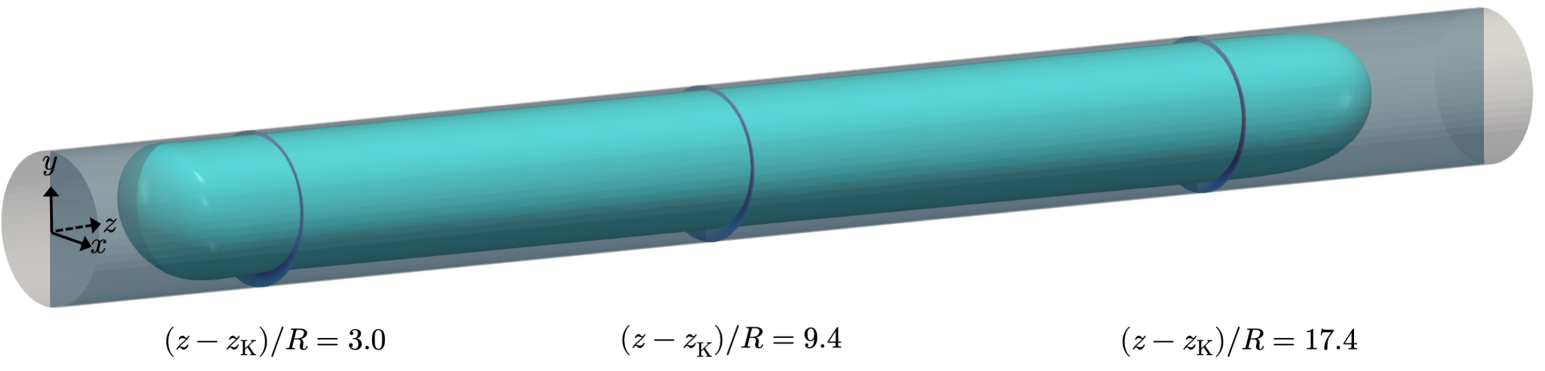

(a)
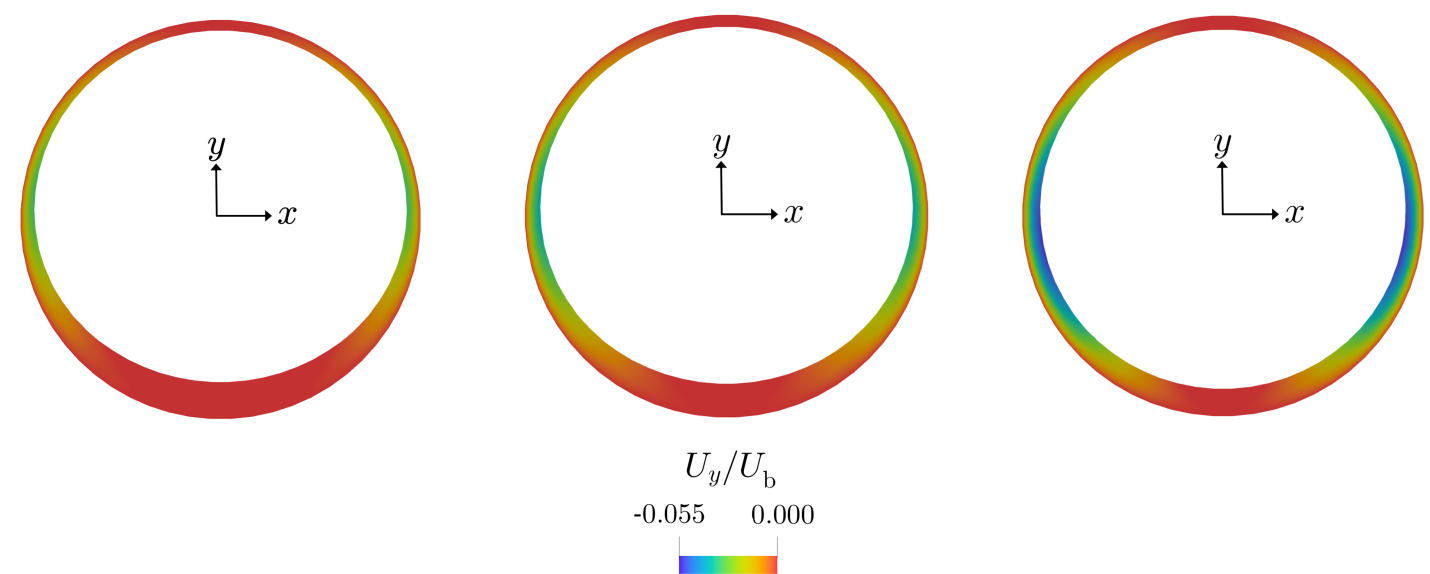

(b) $\left(z-z_{\mathrm{K}}\right) / R=3.0$

(c) $\left(z-z_{\mathrm{K}}\right) / R=9.4$

(d) $\left(z-z_{\mathrm{K}}\right) / R=17.4$

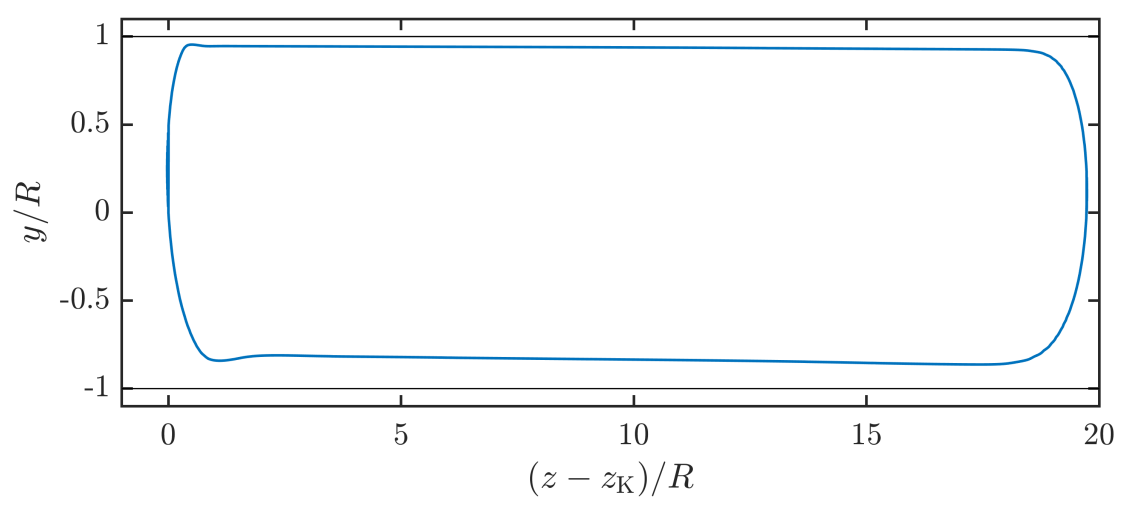

(e)

Figure 4: (a) Profile of an elongated bubble obtained with a numerical simulation for $\mathrm{Ca}_{\mathrm{l}}=0.024$ and $\mathrm{Bo}=0.42\left(\mathrm{We}_{\mathrm{l}} \ll 1\right)$, flow is from left to right; $(\mathrm{b} / \mathrm{d})$ corresponding contour plots of the vertical component of the velocity in the liquid film, at selected cross-sections along the bubble; (e) corresponding bubble profile (not to scale) in the $x=0$ plane.

$H^{-}=0.137$, whilst the inclination angles are $\alpha^{+}=1.2 \times 10^{-3} \mathrm{rad}$ and $\alpha^{-}=2.9 \times 10^{-3} \mathrm{rad}$.

\subsubsection{Bubble shape}

The effect of buoyancy (i.e. increasing Bo) on the bubble shape is studied in Figure 5 . Figure 5 a reveals that, with increasing Bo, the bubble lengthens and moves higher up in the channel, and the top film thins considerably while the bottom film becomes much thicker. Also, the front and rear menisci progressively lose the typical spherical shapes characterising long bubbles propagating in capillary channels, and for $\mathrm{Bo}=3$ it is actually difficult to distinguish between 


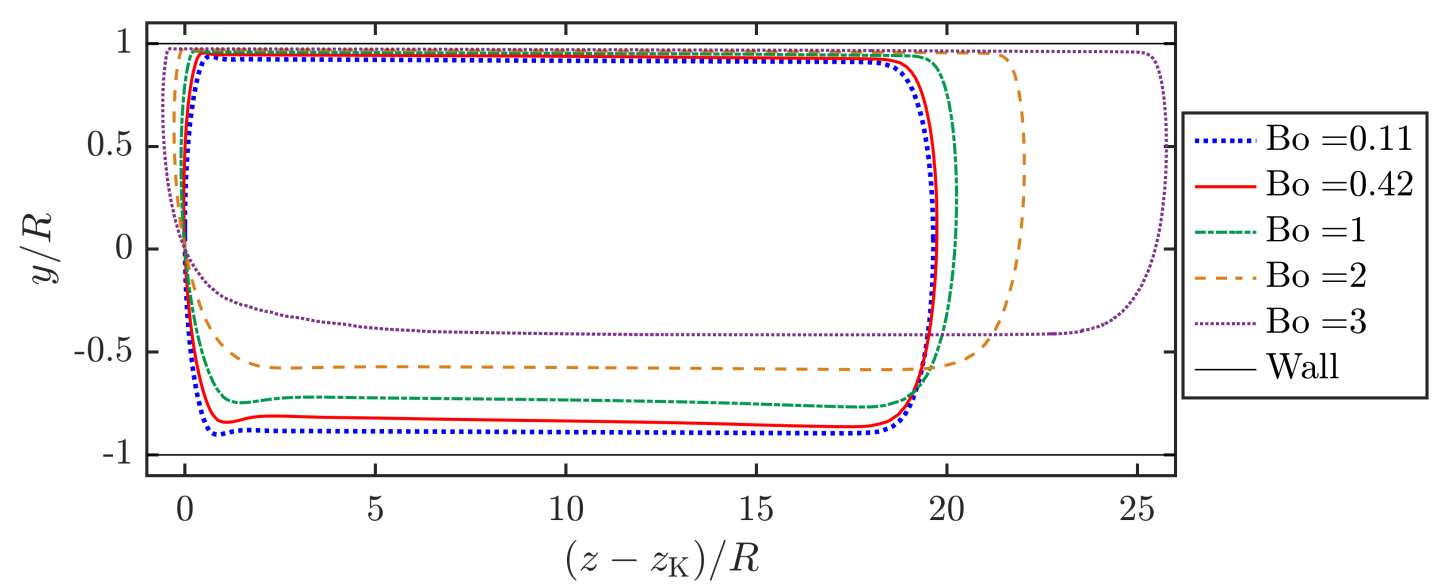

(a)

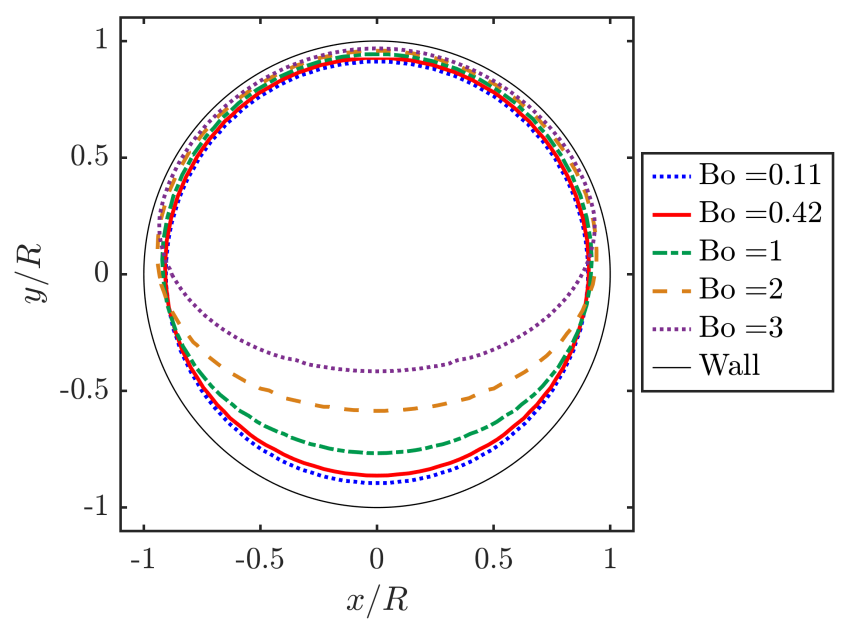

(b)

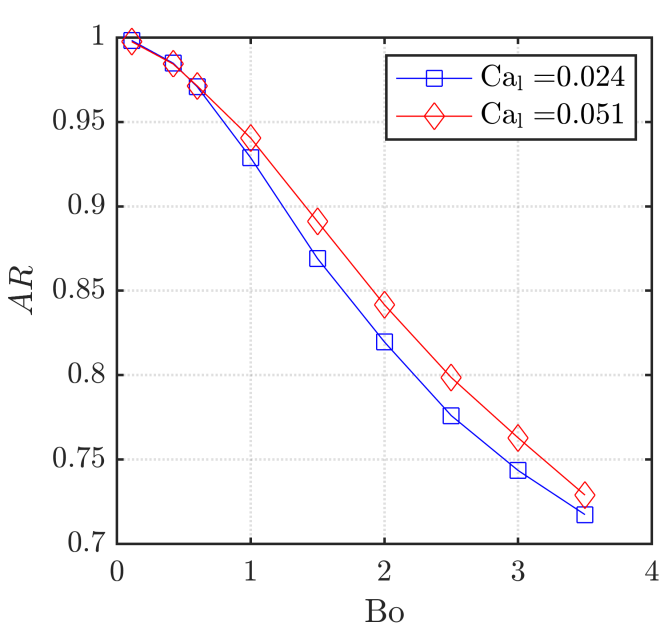

(c)

Figure 5: Systematic analysis of the impact of the Bond number for the negligible inertia case $\left(\mathrm{We}_{1} \ll 1\right)$ on the (a) bubble profile on a vertical centreline plane $(x=0)$, (b) on the bubble cross-sectional shape at the location F (see Fig. 1), and (c) on the bubble cross-sectional aspectratio $A R$ measured at the same location. In (a), flow is from left to right. The capillary number of the liquid in (a) and (b) is $\mathrm{Ca}_{1}=0.024$.

the nose and rear of the bubble, as their shapes look very similar. Figure 5b provides insight into the cross-sectional shape of the bubble when varying the Bond number and emphasises the process of bubble ovalisation. As Bo $\rightarrow 0$, the bubble cross-section tends towards a circular shape, but with increasing Bo it becomes less axisymmetric, and as Bo $>1$ the bubble shape is far from being circular. Also, it can be seen that the upper half of the bubble cross-section remains relatively circular whilst the lower half becomes more oval in shape with increasing Bo. Such deformations of the bubble cross-section due to buoyancy effects were reported in channels with rectangular cross-sections by de Lózar et al. (2008) who attributed this effect to the pressure balance in the liquid film. The hydrostatic pressure in the liquid film increases linearly with distance from the top of the channel, and when the flow is subjected to a transverse gravitational force, this pressure must be balanced with the pressure drop across the interface to maintain a constant pressure inside the bubble, resulting in a decrease in interface curvature with distance from the top of the channel.

The aspect-ratio of the bubble cross-sectional shape, calculated as indicated in Fig. 1, is reported as a function of the Bond number in Fig. $5 \mathrm{c}$. The trends of the deformation of the bubble cross-section are similar for the two values of $\mathrm{Ca}_{1}$ presented. Generally, $A R$ decreases 


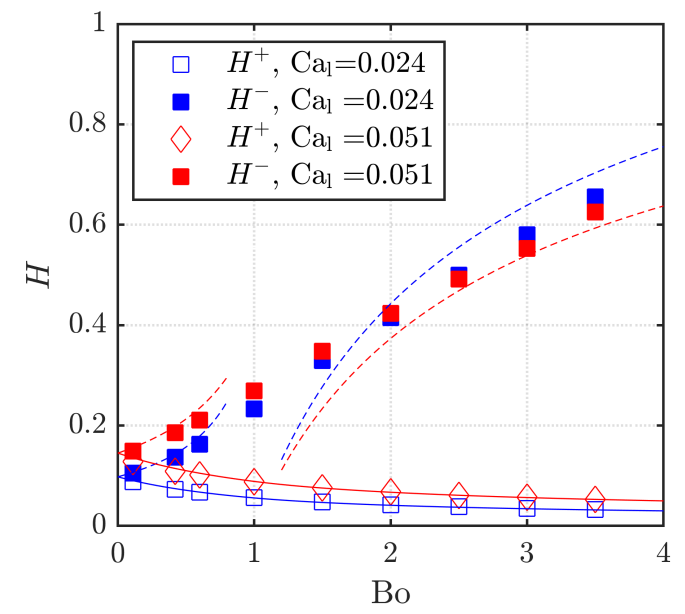

(a)

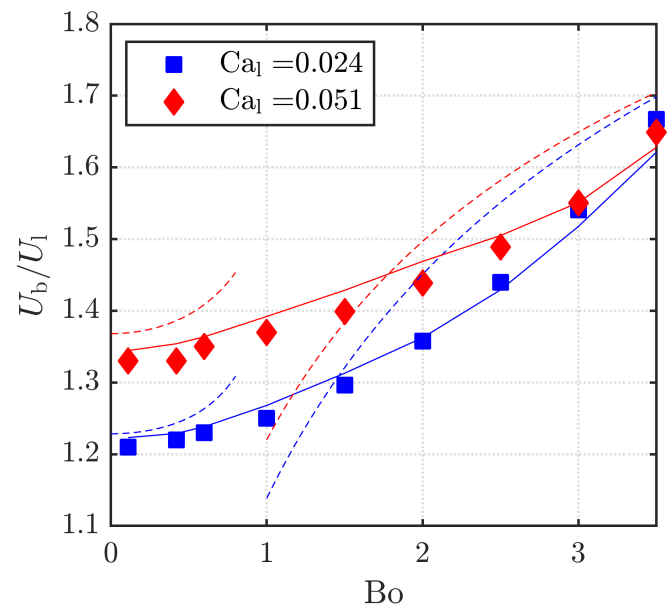

(b)

Figure 6: Impact of the Bond and capillary numbers $\left(\mathrm{We}_{\mathrm{l}} \ll 1\right)$ on the $(\mathrm{a})$ top $\left(H^{+}\right)$and bottom $\left(H^{-}\right)$liquid film thicknesses and (b) bubble advancing speed. In (a), the solid lines represent the predictions for the top film thickness given by Eqs. (13) and (14), while the dashed lines identify the predictions for the bottom thickness obtained with Eqs. (13) and (15). In (b), the lines identify the predictions extracted by applying Eq. (16) with $\bar{H}$ calculated based on the simulation data (solid lines), and with $\bar{H}$ predicted based on Eqs. (13) - 15) for $H^{+}$and $H^{-}$, and Aussillous and Quéré (2000) correlation for $H^{\mathrm{h}}$ (dashed lines).

with increasing Bo, meaning the vertical extent of the bubble cross-section becomes smaller compared to the horizontal extent, and the bubble cross-section becomes less circular in shape. When Bo $>1$, the relative difference of vertical and horizontal extensions of the bubble crosssection rises above $10 \%$. The decreasing trend of $A R$ with Bo is slightly more pronounced for the lower $\mathrm{Ca}_{1}$ case. As derived by Magnini et al. (2019) for the flow of long bubbles in vertical channels, the gravitational force acting on the liquid film scales as Bo/Ca, and therefore the impact of buoyancy is expected to be more significant at lower $\mathrm{Ca}_{1}$, thus explaining the steeper curve at $\mathrm{Ca}_{1}=0.024$ in Fig. $5 \mathrm{c}$.

\subsubsection{Film thickness and bubble speed}

The effect of buoyancy on liquid film thickness and advancing speed of the bubble for flow with negligible inertia are displayed in Fig. 6. All the values of the film thickness in Fig. 6a are measured near the bubble nose, location $\mathrm{F}$ in Fig. 1. As a general trend, $H^{-}$increases with Bo, as observed in Fig. 5 , whilst $H^{+}$decreases with Bo as buoyancy pushes the bubble further up in the channel. The gravitational effects are more pronounced in the bottom film where the film is thicker, due to the combined effect of the rise of the bubble and of the cross-sectional bubble deformation. The film thickness behaviour is similar for the two values of $\mathrm{Ca}_{1}$, although the slope of the $\mathrm{H}^{-}$curve is larger for the smaller $\mathrm{Ca}_{1}$ because the buoyancy effects are more impactful as explained in the previous section, so that eventually when Bo $>2$ the bottom film for $\mathrm{Ca}_{1}=0.024$ is thicker than that for $\mathrm{Ca}_{1}=0.051$. Notably, the top and bottom film thicknesses at the largest Bond numbers tested may differ by more than one order of magnitude. Figure 6a also includes the predictions for the top and bottom film thicknesses estimated with Eqs. (13)- (15). As a first approximation, the input value of $\mathrm{Ca}_{\mathrm{b}}$ in Eqs. (13) - (15) is estimated beforehand by iterating between the Aussillous and Quéré (2000) correlation (Eq. (2)) and the material balance Eq. (16) (with $H$ from Eq. (2)), and thus it does not account for the impact of Bo on the bubble velocity. The model predicts very well the top film thickness in all the ranges of conditions studied, and performs well on the bottom film thickness when Bo $\leq 0.6$ and 
Bo $>1.5$. In the Bo $<1$ range, the mean absolute error on the prediction of top and bottom film thicknesses is of 4.8\%, whereas the model of Atasi et al. (2017) yields an $8 \%$ error. For the entire data set, excluding the bottom film thicknesses for $\mathrm{Bo}=1$ and 1.5 , the error is $4.6 \%$, compared to $20.4 \%$ with Atasi et al. (2017). However, the large error with Atasi et al. (2017) is to be expected since the model is only valid for $\mathrm{Bo}<1$, and does not account for the ovalisation of the bottom of the bubble.

The bubble velocity (Fig. 6b) increases monotonically with the Bond number. As Bo $\rightarrow 0$, $U_{\mathrm{b}}$ converges to the value for negligible gravity, which is an increasing function of $\mathrm{Ca}_{\mathrm{l}}$. However, as Bo increases, the bubble velocity rises owing to the reduced cross-sectional area of the bubble, and the simulation data for different $\mathrm{Ca}_{1}$ seem to converge as Bo $\geq 3$. Notably, for the same liquid flow rate (i.e. same $\mathrm{Ca}_{1}$ ), the bubble travels $50 \%$ faster when $\mathrm{Bo} \geq 3$ compared to the $\mathrm{Bo}=0$ case. The bubble speed trends are closely related to those for the bottom film thickness presented in Fig. 6a. Since bubble speed and cross-sectional area are related via the material balance of the liquid flow, Eq. (16), and at large Bo the extension of the crosssectional area is mostly determined by $H^{-}$(as is clear from Fig. 5b), the trends for $H^{-}$and $U_{\mathrm{b}}$ as a function of $\mathrm{Bo}$ and $\mathrm{Ca}_{1}$ have to be similar. Figure $6 \mathrm{~b}$ includes also two different sets of predictions obtained using Eq. (16). When using simulation data to replace for $H^{+}, H^{-}$and $H^{\mathrm{h}}$ in $\bar{H}=\left(H^{+}+H^{-}+2 H^{\mathrm{h}}\right) / 4$, the bubble velocity is predicted very well, thus emphasising the validity of the material balance also in situations with large buoyancy effects. When evaluating $\bar{H}$ based on the set of Eqs. (13) - 15) for $H^{+}$and $H^{-}$, and on Eq. (2) for $H^{\mathrm{h}}$, the predictions are still relatively good except when $\mathrm{Bo} \approx 1$.

Additional data illustrating the streamwise pressure profiles along the channel for this set of Bond and capillary numbers are included as Supplementary Material.

\subsubsection{Liquid film drainage velocity and bubble inclination angle}

Leung et al. (2012) and Atasi et al. (2017) have demonstrated that the inclination of the bubble originates from the draining flow from the top to the bottom of the channel, and thus the

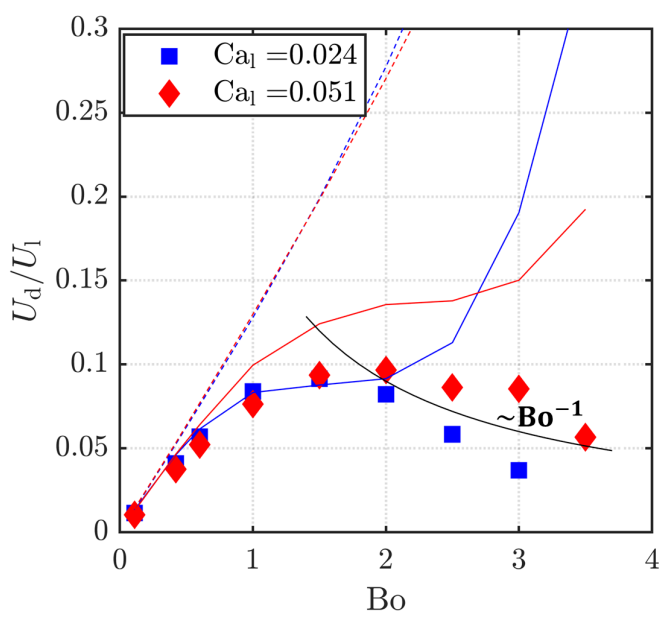

(a)

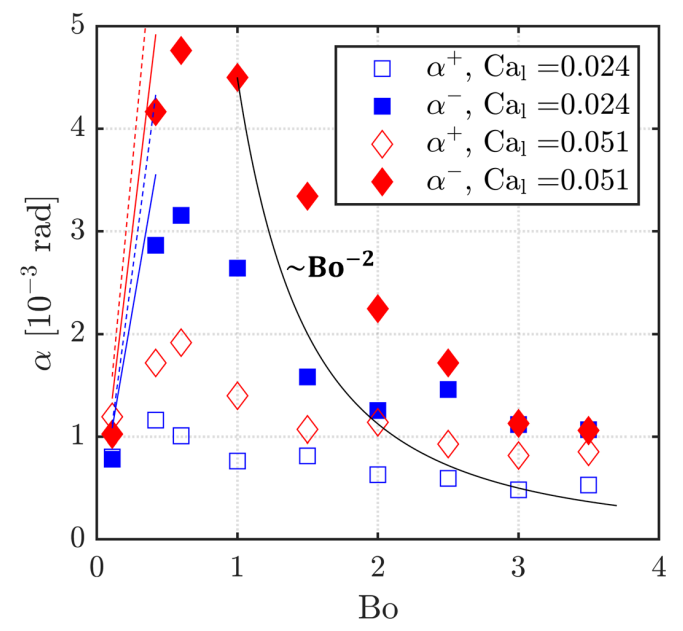

(b)

Figure 7: Impact of the Bond and capillary numbers $\left(\mathrm{We}_{1} \ll 1\right)$ on the $(\mathrm{a})$ average liquid film drainage velocity and (b) top $\left(\alpha^{+}\right)$and bottom $\left(\alpha^{-}\right)$bubble inclination angles. Red and blue lines identify the predictions obtained using (a) Eq. (18) with $\sin \theta=1$, and (b) Eq. (6), with $H^{\mathrm{h}}$ based on simulation data (solid lines) or Aussillous and Quéré (2000) correlation (dashed lines). The black solid lines identify the scaling laws extracted from the analysis of the minimum film thickness trends in Fig. 8 . 
trends of drainage velocity and bubble inclination angle are expected to be correlated. This is confirmed by the data obtained with our numerical simulations, which are presented in Fig. 7 . The drainage velocity $U_{\mathrm{d}}$ is calculated on the tube cross-section at the location $\mathrm{F}$, by taking the mean value of the $y$-component of the velocity in the liquid film, $U_{y}$, in the horizontal plane where $E_{x}$ and $H^{\mathrm{h}}$ are measured (see Fig. 1). The values of bubble inclination reported in Fig. $7 \mathrm{~b}$ are measured by taking the average slope of the top and bottom profiles of the bubble (on the plane $x=0$ ), in a region $4 D$ long at the bubble centre. The data in Fig. 7 show that both drainage velocity and bubble inclination initially increase with the Bond number, owing to the initiation of draining flows as the liquid in the film begins moving downward under the effect of gravity. Both $U_{d}$ and $\alpha$ increase linearly with $\mathrm{Bo}$ when $\mathrm{Bo}<1$, which is consistent with the scalings $U_{d} \sim$ Bo $H^{2}$ and $\alpha \sim$ Bo $H^{3}$ arising, respectively, from Eqs. (18) and (6), with $H \equiv H^{\mathrm{h}}$ being approximately constant in this regime (see cross-sectional profiles in Fig. 5b).

When Bo $>1$, the simulation data show that both $U_{d}$ and $\alpha$ drop as the Bond number is further increased; this declining trend at large Bo was not observed in previous studies, which were limited to the Bo $<1$ regime. Figure $5 \mathrm{~b}$ reveals that, as Bo $>1$, the bubble cross-section becomes oval and the film thickness on the top half of the bubble reduces considerably. In this regime, the $\sim H^{2}$ and $\sim H^{3}$ terms in Eqs. (18) and (6) take over and the drainage flow rate reduces because there is very little liquid available to drain from the top film region. Figure 7 includes the predictions of $U_{d}$ and $\alpha$ obtained using the existing theory based on Eqs. (18) and (6). While the theory works well as long as the bubble cross-section is circular $(\mathrm{Bo}<1)$, it fails when Bo $>1$ because the bubble cross-section becomes oval and $H^{\mathrm{h}}$ is no longer representative of the actual gap available for the liquid to flow between the bubble interface and the wall, which is much smaller. At a first glance, the top film thickness $H^{+}$may seem a more suitable parameter to calculate the drainage flow rate. However, Eq. (14) yields $H^{+} \sim 1 / \sqrt{\mathrm{Bo}}$ as Bo $\gg 1$. This would give $U_{d} \sim$ Bo $\left(H^{+}\right)^{2} \sim 1$, i.e. constant $U_{d}$ at large Bo, which does not match the present data. Hence, the gap available for the draining liquid to flow must decrease as Bo increases, at a faster rate than $H^{+}$does.

In order to investigate the perimetral evolution of the liquid film more in detail, Fig. $8 \mathrm{a}$

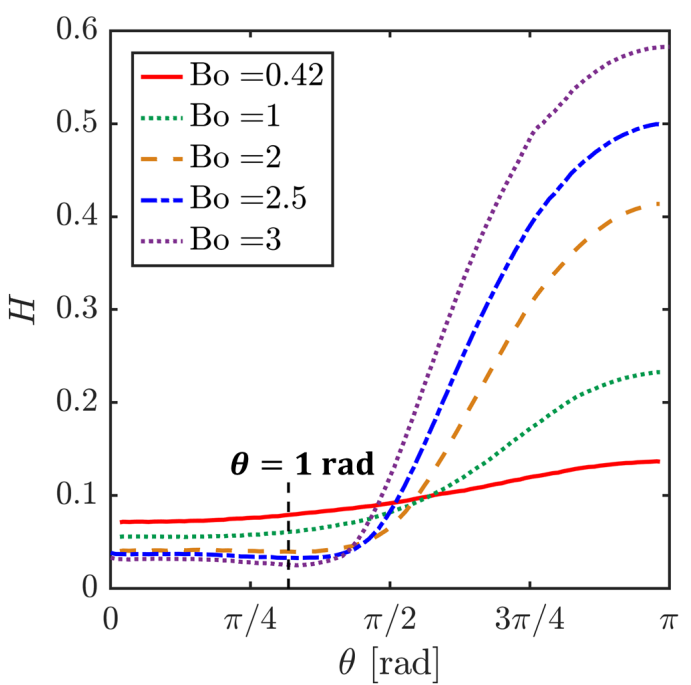

(a)

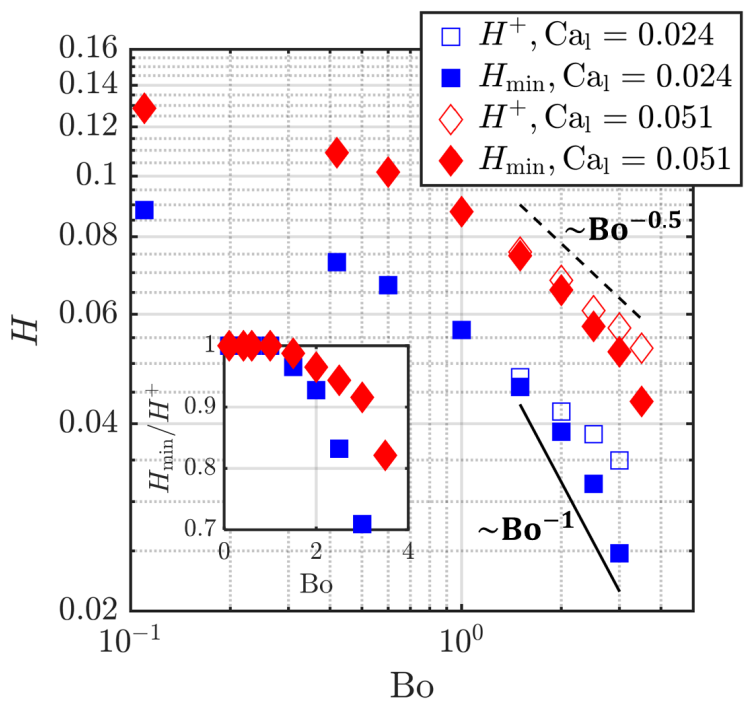

(b)

Figure 8: (a) Circumferential evolution of the liquid film thickness on the channel cross-section at the location $\mathrm{F}$ for $\mathrm{Ca}_{1}=0.024$ and different Bond numbers $\left(\mathrm{We}_{\mathrm{l}} \ll 1\right.$ ), and (b) top and minimum liquid film thickness at $\mathrm{F}$ as a function of Bond and capillary numbers. In (a), $\theta=0$ corresponds to the top of the channel, see Fig. 1 . 
displays the cross-sectional film thickness for the interface profiles depicted in Fig. 5b, thus $\mathrm{Ca}_{1}=0.024$, plotted as a function of the circumferential coordinate $\theta$. It can be seen that, as the Bond number increases above 1 , the top $(\theta=0)$ and bottom $(\theta=\pi)$ film thicknesses diverge. This leads to the creation of a constriction in the film, at the matching point between the thin film at the top and the thick liquid lobe at the bottom. This constriction, whose circumferential location is constant along the bubble body and converges to $\theta=1 \mathrm{rad}$ as Bo increases, identifies a minimum value of the liquid film thickness $H_{\min }=h_{\min } / R$. Note that a liquid film constriction with very similar morphological features has been reported for the film profile around long bubbles translating in square channels, at the matching point between the thin film at the cross-section centre and the thick liquid lobes at the corners (Wong et al. 1995. Hazel and Heil 2002 Magnini and Matar 2020). Figure 8b displays the values of both $\mathrm{H}^{+}$and $H_{\min }$ measured at the location $\mathrm{F}$ and indicates that the deviation between $\mathrm{H}^{+}$and $H_{\text {min }}$ increases with $\mathrm{Bo}$, with $H^{+}$obeying a scaling law $H^{+} \sim \mathrm{Bo}^{-1 / 2}$ (in agreement with Eq. (14)), whereas $H_{\text {min }} \sim \mathrm{Bo}^{-1}$. Interestingly, these two scalings yield $H_{\min } \sim\left(H^{+}\right)^{2}$, which is the same law obtained theoretically by Wong et al. (1995) for flow in square channels, where $H \sim \mathrm{Ca}^{2 / 3}$ at the centre and $H_{\min } \sim \mathrm{Ca}^{4 / 3}$ at the constriction. Using the minimum film thickness as the characteristic length scale for evaluating the draining flow rate, the scaling laws $U_{d} \sim \mathrm{Bo}\left(H_{\min }\right)^{2} \sim 1 / \mathrm{Bo}$ and $\alpha \sim \mathrm{Bo}\left(H_{\min }\right)^{3} \sim 1 / \mathrm{Bo}^{2}$ are obtained. These laws capture well the trends of the average drainage velocity and bubble inclination angle for $\mathrm{Bo}>1$, as indicated in Fig. 7.

Figure $7 \mathrm{~b}$ shows also that the inclination angle of the bubble at the top $\alpha^{+}$is systematically smaller than that at the bottom $\alpha^{-}$, as was observed experimentally by Atasi et al. (2017), and that $\alpha$ increases with the capillary number, due to the development of thicker films which are subject to more copious draining flows.

\subsection{Inertial effects}

A parametric study of the effect of inertia on elongated bubble flows with non-negligible buoyancy effects is presented, for a range of values of $\mathrm{Bo}, \mathrm{Ca}_{1}$ and $\mathrm{Re}_{1}$. Behaviour as a function of $\mathrm{Re}_{1}$ was found to be similar for different values of $\mathrm{Ca}_{1}$ so one representative case, $\mathrm{Ca}_{1}=0.024$, is discussed in this section. A further case, $\mathrm{Ca}_{1}=0.051$, is provided as Supplementary Material.

\subsubsection{Bubble shape}

The impact of inertial and buoyancy forces on the bubble profile can be studied by means of Figure 9, which reports the geometrical features of the bubbles for a fixed value of $\mathrm{Ca}_{1}=0.024$, two values of the Bond number, $\mathrm{Bo}=0.42$ and 1 , and the range of Reynolds numbers $\operatorname{Re}_{1}=$ $1-750$. Figure 9a shows that the bubble nose elongates and its cross-section shrinks upon increase of the Reynolds number, which is in line with the literature concerning flows in the absence of buoyancy (Heil 2001; Kreutzer et al. 2005). Interestingly, as the bubble cross-section reduces, the liquid film around it does not thicken uniformly around the tube perimeter, but only the bottom film gets substantially thicker, while the top film thickness is unchanged, such that inertial effects further elevate the bubble above the channel axis. Inertial effects are known for being responsible for the appearance of interfacial undulations near the tail of long bubbles when $\mathrm{We}_{\mathrm{l}} \geq 1$ (Magnini et al. 2017b). It can be seen in Fig. 9a that these appear only on the bottom profile at intermediate values of $\mathrm{Re}_{\mathrm{l}}$, whereas the profile on the top stays smooth. This can be explained by considering that the impact of inertia on the free-surface profile is better described by the Reynolds number calculated based on the actual film thickness, rather than the channel diameter which has little importance on the film dynamics. Since the bottom film is more than twice thicker than the top film, its Reynolds number is larger and undulations are expected to appear earlier. We observe that the interfacial undulations become less evident as $\mathrm{Re}_{\mathrm{l}}$ is increased to $\mathrm{Re}_{\mathrm{l}}=750$ and the bubble dynamics becomes time-dependent, which is 


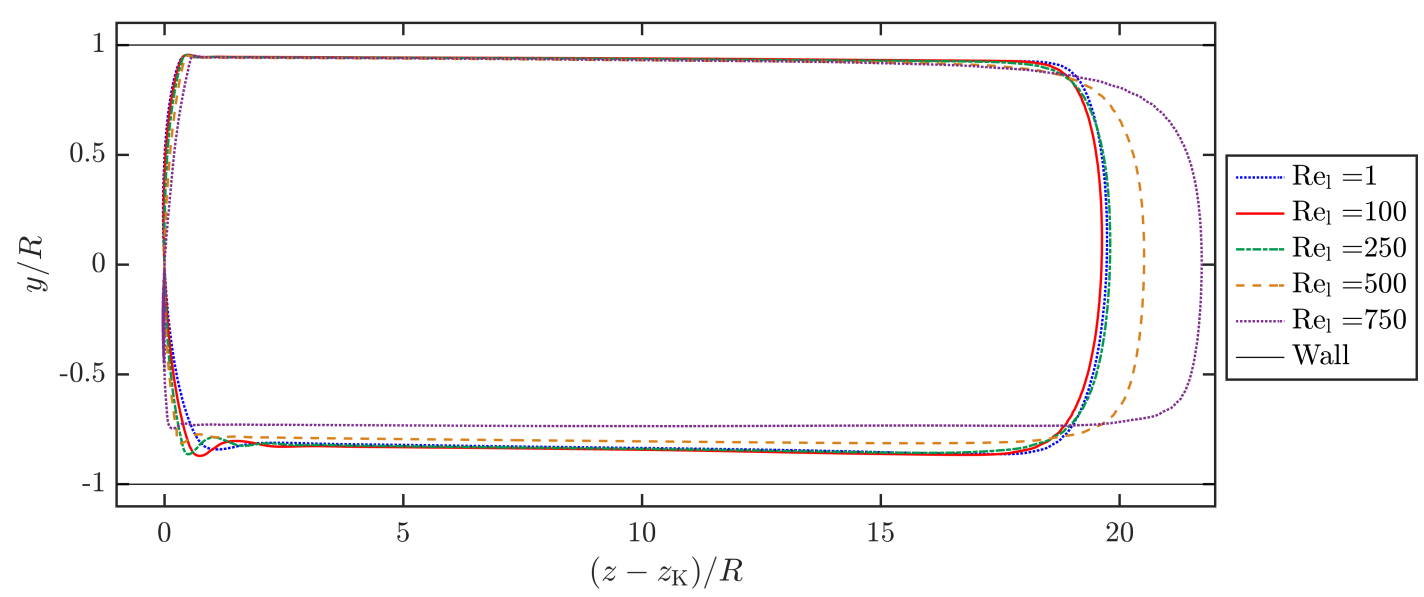

(a)

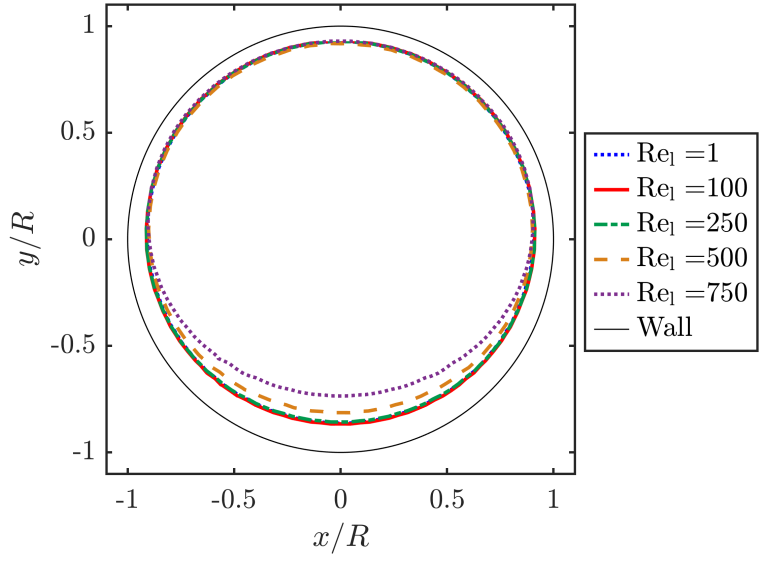

(b)

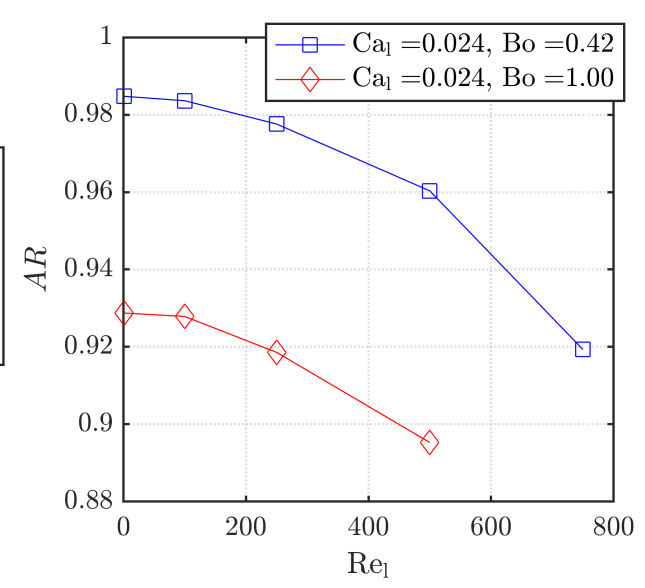

(c)

Figure 9: Systematic analysis of the impact of the Reynolds number on the (a) bubble profile on a vertical centreline plane $(x=0)$, (b) on the bubble cross-sectional shape at the location $\mathrm{F}$, and (c) on the bubble cross-sectional aspect-ratio measured at the same location. In (a) and (b), conditions are $\mathrm{Ca}_{1}=0.024$ and $\mathrm{Bo}=0.42$.

consistent with the large Weber number of the flow, $\mathrm{We}_{1}=18$ (Magnini et al. 2017b). The bubble profiles on the channel cross-section depicted in Fig. 9b show that the increase of the Reynolds number is responsible for a more apparent bubble ovalisation, and thus the crosssectional bubble aspect-ratio decreases monotonically as $\mathrm{Re}_{\mathrm{l}}$ increases, as shown in Fig. $9 \mathrm{c}$ for two different values of the Bond number.

\subsubsection{Film thickness and inclination angle}

Figure 10 shows the combined impact of inertia and buoyancy on the liquid film thickness and bubble inclination angle in elongated bubble flow. The inclination angles have been assessed using different lengths of the central region of the bubble to ensure the reported values are independent of nose or tail behaviour. It can been seen from Fig. 10a cc that the bottom film thickness increases with Bo as discussed in Section 4.2, and it increases with $\mathrm{Re}_{\mathrm{l}}$ as already revealed by the profiles in Fig. 9. The impact of $\mathrm{Re}_{1}$ on $H^{-}$is more apparent than usually observed for the Bo $=0$ case because $H^{-}$is already substantially thicker than the gravity-free case at the same $\mathrm{Re}_{\mathrm{l}}$, and hence the thicker film is more sensitive to inertial forces for the reasons explained in the previous section. Accordingly, the top film thickness is only weakly dependent on $\mathrm{Re}_{\mathrm{l}}$ in the range of Reynolds numbers studied, because this thinner film is less sensitive to 


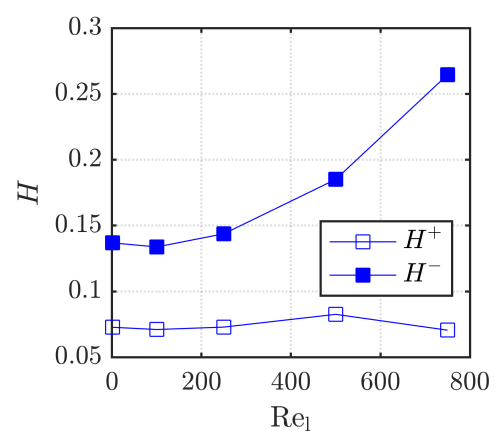

(a) $\mathrm{Bo}=0.42$

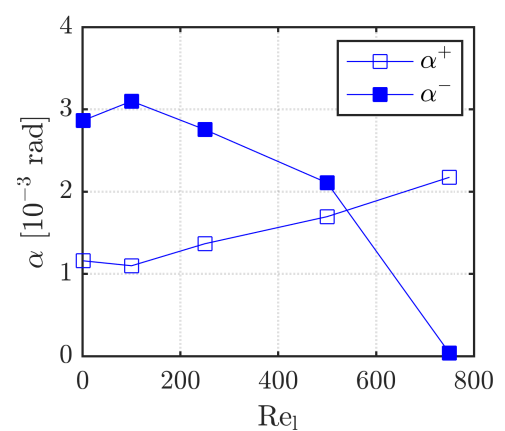

(d) $\mathrm{Bo}=0.42$

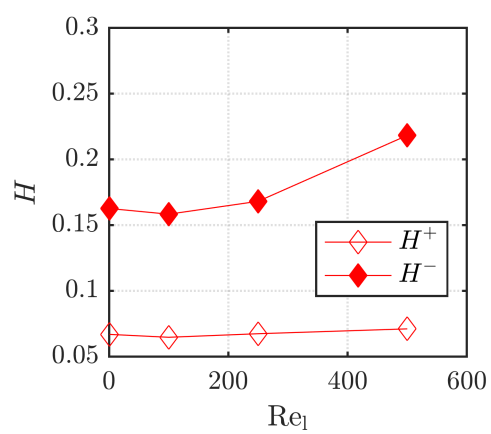

(b) $\mathrm{Bo}=0.60$

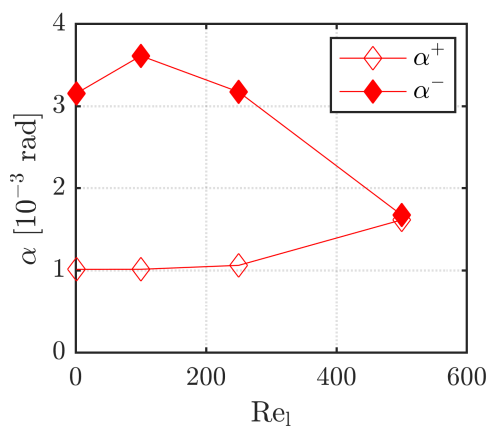

(e) $\mathrm{Bo}=0.60$

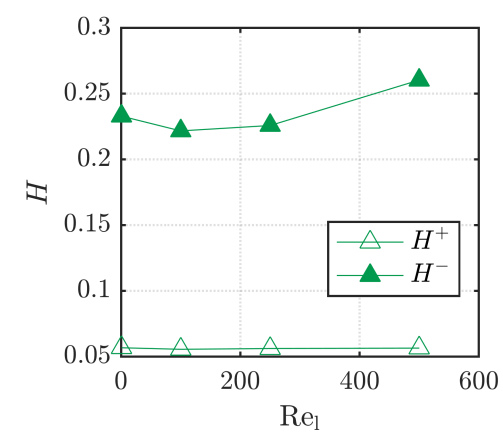

(c) $\mathrm{Bo}=1.00$

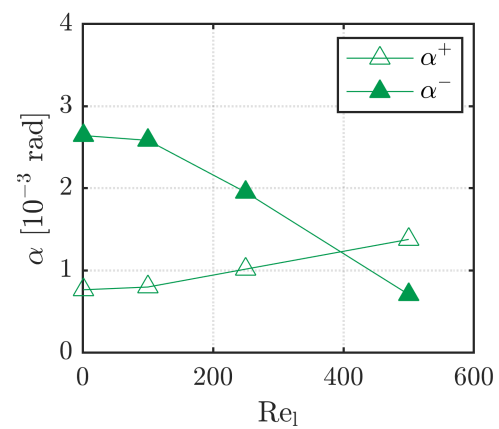

(f) $\mathrm{Bo}=1.00$

Figure 10: Systematic analysis of the effect of the Reynolds number on the (atc) top and bottom liquid film thickness, and $(\mathrm{d}+\mathrm{f})$ on the top and bottom bubble inclination. The liquid capillary number is fixed to $\mathrm{Ca}_{1}=0.024$.

the inertial effects until much larger values of $\mathrm{Re}_{\mathrm{l}}$ are achieved.

The bubble inclinations plotted in Fig. 10d ff show that generally $\alpha^{-}$decreases and $\alpha^{+}$ increases with increasing $\mathrm{Re}_{\mathrm{l}}$, although the effect is again more pronounced at the bottom of the bubble. This effect could be seen also in Fig. 9a, where the bottom profiles of the bubble become less inclined as $\mathrm{Re}_{1}$ increases. The same trends occur for the larger capillary number tested, see the data in the Supplementary Material. Figure 9b shows that the top half of the bubble cross-section changes very little with $\mathrm{Re}_{\mathrm{l}}$, so that the top $\left(H^{+}\right)$and horizontal $\left(H^{\mathrm{h}}\right)$ film thicknesses are essentially constant, thus suggesting that the flow rate of the draining flows is constant with $\mathrm{Re}_{\mathrm{l}}$. However, since the bottom film becomes substantially thicker and thus it contains more liquid as $\mathrm{Re}_{\mathrm{l}}$ increases, it is expected that the incoming flow rate from the thin film induces a smaller relative change on the bottom film volume, and thus on $\alpha^{-}$.

\section{Conclusions}

A systematic study of the effects of buoyancy and inertia on the flow of elongated bubbles in horizontal channels of circular cross-section has been performed. Results are reported from direct numerical simulations with capillary, Bond, and Reynolds numbers in the ranges $0.024<$ $\mathrm{Ca}_{1}<0.051,0.11<\mathrm{Bo}<3.5$, and $1<\mathrm{Re}_{\mathrm{l}}<750$. The simulations were conducted by adopting the Volume-Of-Fluid method as included in the interFoam solver of OpenFOAM. This work demonstrates that the cross-section of the bubble undergoes large deformations as Bo $>1$, with the circular shape becoming progressively more oval, and the width and height of the bubble cross-section differing by more than $25 \%$ as Bo $\geq 3$.

Due to buoyancy, the bubble elevates above the channel centreline. The top and bottom film thicknesses diverge as Bo $>0$ and their difference grows to one order of magnitude and 
above as $\mathrm{Bo}>3$, with the bubble travelling $50 \%$ faster compared to the gravity-free case at the same liquid flow rate. We observe bubble inclination with respect to the channel axis, with the tail being higher than the nose, and we show that this effect is induced by the downward liquid flow that drains liquid from the top to the bottom film region. However, the bubble inclination does not increase monotonically with the Bond number, due to the formation of a localised constriction in the thin film on the top half of the bubble, whose thickness decreases as $\sim 1$ Bo. As the top liquid film becomes thinner, viscous drag overcomes gravity, the flow rate of the draining flow reduces, and thus the bubble inclination becomes less apparent at the largest Bond numbers tested. Inertial forces tend to shrink the bubble cross-section and further lift the bubble above the channel centreline, so that the bottom film thickness increases significantly with the Reynolds number whereas the top film thickness is less sensitive to it.

We propose new prediction models for the top and bottom film thicknesses and the bubble speed which incorporate the effects of the capillary and Bond numbers. These models compare well with our simulation data for Bo up to 3.5, although the convergence of the bottom film thickness predictions as Bo $\rightarrow 1$ can be improved. The existing models for the liquid film draining flows and the bubble inclination perform well as long as Bo $<1$ and the bubble crosssection remains circular, but the bubble deformation cannot be neglected in order to extend the predictive capability of these models to Bond numbers larger than unity.

\section{Acknowledgements}

O. K. M. acknowledges funding from PETRONAS and the Royal Academy of Engineering for a Research Chair in Multiphase Fluid Dynamics. This work is also supported by the UK Engineering \& Physical Sciences Research Council (EPSRC), through the PREMIERE (EP/T000414/1) Programme Grant and by the Department for International Development (DFID) through the Royal Society-DFID Africa Capacity Building Initiative. We also acknowledge use of the HPC facilities provided by the Research Computing Service (RCS) of Imperial College London for the computing time.

\section{Supplementary material}

Supplementary material associated with this article is included with the submission. This includes: results of a mesh independence study; additional data illustrating pressure profiles along the channel in the $\mathrm{We}_{1} \ll 1$ regime to complement the results presented in Section 4.2 ; data for the dynamics of bubbles in the $\mathrm{We}_{1} \gg 1$ regime, with $\mathrm{Ca}_{1}=0.051$, to complement the results presented in Section 4.3 .

\section{References}

Atasi, O., Khodaparast, S., Scheid, B., and Stone, H. A. (2017). "Effect of buoyancy on the motion of long bubbles in horizontal tubes". Physical Review Fluids 2 (9), 094304. DOI: 10.1103/PhysRevFluids.2.094304.

Aussillous, P. and Quéré, D. (2000). "Quick deposition of a fluid on the wall of a tube". Physics of Fluids 12 (10), 2367-2371. DOI: 10.1063/1.1289396.

Brackbill, J., Kothe, D., and Zemach, C. (1992). "A continuum method for modeling surface tension". Journal of Computational Physics 100 (2), 335-354. DOI: 10.1016/0021-9991(92) 90240-Y.

Bretherton, F. P. (1961). "The motion of long bubbles in tubes". Journal of Fluid Mechanics 10 (02), 166-188. DOI: $10.1017 /$ S0022112061000160.

de Lózar, A., Juel, A., and Hazel, A. L. (2008). "The steady propagation of an air finger into a rectangular tube". Journal of Fluid Mechanics 614, 173-195. DOI:10.1017/S0022112008003455 
Deshpande, S. S., Anumolu, L., and Trujillo, M. F. (2012). "Evaluating the performance of the two-phase flow solver interFoam". Computational Science $\&$ Discovery 5 (1), 014016. DoI: 10.1088/1749-4699/5/1/014016

Dirker, J., Juggurnath, D., Kaya, A., Osowade, E. A., Simpson, M., Lecompte, S., Abadi, S. M. R., Voulgaropoulos, V., Adelaja, A. O., Dauhoo, M. Z., Khoodaruth, A., Obayopo, S. O., Olakoyejo, O. T., Elahee, M. K., De Paepe, M., Meyer, J. P., and Markides, C. N. (2019). "Thermal energy processes in direct steam generation solar systems: Boiling, condensation and energy storage". Frontiers in Energy Research 6, 147. DOI: 10.3389/fenrg. 2018.00147.

Ferrari, A., Magnini, M., and Thome, J. R. (2017). "A Flexible Coupled Level Set and Volume of Fluid (flexCLV) method to simulate microscale two-phase flow in non-uniform and unstructured meshes". International Journal of Multiphase Flow 91, 276-295. DOI: 10.1016/ J.IJMULTIPHASEFLOW.2017.01.017.

Gupta, R., Fletcher, D. F., and Haynes, B. S. (2009). "On the CFD modelling of Taylor flow in microchannels". Chemical Engineering Science 64 (12), 2941-2950. DOI: 10.1016/J . CES . 2009.03 .018

Han, Y. and Shikazono, N. (2009). "Measurement of the liquid film thickness in micro tube slug flow". International Journal of Heat and Fluid Flow 30 (5), 842-853. DOI: 10.1016/j. ijheatfluidflow.2009.02.019.

Hazel, A. L. and Heil, M. (2002). "The steady propagation of a semi-infinite bubble into a tube of elliptical or rectangular cross-section". J. Fluid Mech. 470, 91-114. DOI: 10.1017/ S0022112002001830.

Heil, M. (2001). "Finite Reynolds number effects in the Bretherton problem". Physics of Fluids 13 (9), 2517-2521. DOI: $10.1063 / 1.1389861$.

Hirt, C. W. and Nichols, B. D. (1981). "Volume of fluid (VOF) method for the dynamics of free boundaries". Journal of Computational Physics 39 (1), 201-225. DOI: 10.1016/00219991(81) 90145-5.

Issa, R. I. (1986). "Solution of the implicitly discretised fluid flow equations by operatorsplitting". Journal of Computational Physics 62 (1), 40-65. DOI: 10.1016/0021-9991(86) 90099-9.

Jensen, M. H., Libchaber, A., Pelce, P., and Zocchi, G. (1987). "Effect of gravity on the SaffmanTaylor meniscus: Theory and experiments". Physical Review A 35 (5), 2221-2227. DOI: 10. 1103/physreva.35.2221.

Karayiannis, T. G. and Mahmoud, M. M. (2017). "Flow boiling in microchannels: Fundamentals and applications". Applied Thermal Engineering 115, 1372-1397. DOI: $10.1016 / \mathrm{j}$. applthermaleng.2016.08.063.

Khodaparast, S., Magnini, M., Borhani, N., and Thome, J. R. (2015). "Dynamics of isolated confined air bubbles in liquid flows through circular microchannels: an experimental and numerical study". Microfluidics and Nanofluidics 19, 209-234. DOI: 10.1007/s10404-0151566-4.

Kreutzer, M. T., Kapteijn, F., Moulijn, J. A., Kleijn, C. R., and Heiszwolf, J. J. (2005). "Inertial and interfacial effects on pressure drop of Taylor flow in capillaries". AIChE Journal 51 (9), 2428-2440. DOI: 10.1002/aic.10495.

Leung, S. S., Gupta, R., Fletcher, D. F., and Haynes, B. S. (2012). "Gravitational effect on Taylor flow in horizontal microchannels". Chemical Engineering Science 69 (1), 553-564. DOI: 10.1016/J.CES.2011.11.016

Magnini, M., Beisel, A. M., Ferrari, A., and Thome, J. R. (2017a). "Pore-scale analysis of the minimum liquid film thickness around elongated bubbles in confined gas-liquid flows". Advances in Water Resources 109, 84-93. DOI: 10.1016/j.advwatres.2017.08.020. 
Magnini, M., Ferrari, A., Thome, J. R., and Stone, H. A. (2017b). "Undulations on the surface of elongated bubbles in confined gas-liquid flows". Physical Review Fluids 2 (8), 084001. DOI: 10.1103/PhysRevFluids.2.084001.

Magnini, M., Khodaparast, S., Matar, O. K., Stone, H. A., and Thome, J. R. (2019). "Dynamics of long gas bubbles rising in a vertical tube in a cocurrent liquid flow". Physical Review Fluids 4 (2), 023601. DOI: 10.1103/PhysRevFluids.4.023601.

Magnini, M. and Matar, O. K. (2020). "Morphology of long gas bubbles propagating in square capillaries". International Journal of Multiphase Flow 120, 103353. DOI: 10.1016/j . ijmultiphaseflow . 2020.103353.

Taylor, G. I. (1961). "Deposition of a viscous fluid on the wall of a tube". Journal of Fluid Mechanics 10 (2), 161-165. DOI: 10.1017/S0022112061000159.

Wong, H., Radke, C. J., and Morris, S. (1995). "The motion of long bubbles in polygonal capillaries. Part 1. Thin films". J. Fluid Mech. 292, 71-94. DOI: 10.1017/S0022112095001443. 